\title{
A new genus of Baetidae (Insecta, Ephemeroptera) from Southeast Asia
}

\author{
Thomas KALTENBACH ${ }^{1, *}$, Jhoana M. GARCES ${ }^{2} \&$ Jean-Luc GATTOLLIAT ${ }^{3}$ \\ ${ }^{1,3}$ Museum of Zoology, Palais de Rumine, Place Riponne 6, CH-1005 Lausanne, Switzerland; \\ and University of Lausanne (UNIL), Department of Ecology and Evolution, \\ CH-1015 Lausanne, Switzerland. \\ ${ }^{2}$ Department of Biology, School of Science and Engineering, Ateneo de Manila University, \\ Quezon City, 1108 Metro Manila, Philippines. \\ *Corresponding author: thomas.kaltenbach@bluewin.ch \\ ${ }^{2}$ Email: jhoana.garces@obf.ateneo.edu \\ ${ }^{3}$ Email: jean-luc.gattolliat@vd.ch \\ ${ }^{1}$ urn:1sid:zoobank.org:author:88232ABC-223C-4503-98DD-D563BEDFAF6B
${ }^{2}$ urn:1sid:zoobank.org:author:BB742FEE-6EA4-4910-98BA-E868DD5400D6
${ }^{3}$ urn:1sid:zoobank.org:author:9F2CBF71-33B8-4CD7-88D3-85D7E528AEA5
}

\begin{abstract}
A new genus of Baetidae is described from Southeast Asia, Procerobaetis gen. nov. It has a wide distribution reaching from Indonesia (Sumatra) to the Philippines. Two new species are described from Indonesia, P. leptobranchius gen. et sp. nov. and P. petersorum gen. et sp. nov., and one new species from the Philippines, $P$. freitagi gen. et sp. nov. Procerobaetis gen. nov. is characterized by having seven pairs of elongate, apically pointed gills. At least gills I and II are very slender with strongly extended points, which is unique in Baetidae. Similar gills were described from Leptophlebiidae. Procerobaetis gen. nov. is further characterized by having long, slender legs with extended, slender and slightly bent claws. The antennae posess remarkable spines at the outer, lateral margin, which are maximally developed on segments IX-XI of the flagellum. No spines are present on the posterior margins of abdominal tergites I-VI. COI sequences were obtained from all three of the new species. The genetic distances (Kimura 2-parameter) between these species are between 13\% and 20\%. Very limited genetic distances of $0 \%$ to $1 \%$ were found between specimens of the same species. The occurrence of two different species in the same area of Sumatra is discussed.
\end{abstract}

Keywords. COI, Indonesia, morphology, Philippines, Procerobaetis.

Kaltenbach T., Garces J.M. \& Gattolliat J.-L. 2020. A new genus of Baetidae (Insecta, Ephemeroptera) from Southeast Asia. European Journal of Taxonomy 612: 1-32. https://doi.org/10.5852/ejt.2020.612

\section{Introduction}

The family Baetidae has the highest species diversity among mayflies, comprising 1070 species in 110 genera (Sartori \& Brittain 2015; Jacobus et al. 2019), which is about 30\% of all mayfly species worldwide (Jacobus et al. 2019). They have a cosmopolitan distribution, except in Antarctica and New Zealand. 
Investigations of the molecular phylogeny of the Order Ephemeroptera have revealed the relatively primitive status of the family (Ogden \& Whiting 2005; Ogden et al. 2009). The generic diversity of Baetidae is the highest in the Afrotropical (about 40 genera), followed by the Neotropical (about 27 genera) and Oriental (26 genera) realms and finally the Nearctic (20 genera), Palaearctic (17 genera) and the Australasian (about 12 genera) realms (Gattolliat \& Nieto 2009; Gattolliat 2012). The number for the Oriental realm seems to be rather low considering that regions with potentially high diversities (i.e., the Indian subcontinent and Southeast Asia) belong to this realm. For example, intensive sampling in an area of only $85 \mathrm{~km}^{2}$ on the island Borneo (Indonesia, East Kalimantan) revealed the occurence of 12 different genera of Baetidae, some of them undescribed (Sartori et al. 2003). This could be attributed to a relatively low mayfly research output in the Oriental realm over a long period. Apart from Sri Lanka, Taiwan and Hong Kong, most of the Oriental realm has remained very poorly known (Gattolliat \& Nieto $2009)$, even to date. The last two new genera of Baetidae from the Oriental region were described in 2012 from Borneo (Gattolliat 2012).

Here we describe a new genus from Southeast Asia, based on material collected by Morgan Gueuning (University of Neuchatel, Switzerland; Gueuning et al. 2017) in Indonesia and on the collection stored in the Biodiversity Laboratory headed by Hendrik Freitag (Ateneo de Manila University, Philippines) in the Philippines.

Indonesia is an immense archipelago of more than 18000 islands extending over $5000 \mathrm{~km}$ from $95^{\circ} \mathrm{E}$ to $141^{\circ} \mathrm{E}$ and from $6^{\circ} \mathrm{N}$ to $11^{\circ} \mathrm{S}$. It is one of the most biologically rich countries in the world. The high levels of species richness and endemism are mainly attributable to a complex geological history, that brought together two different biological realms (the Oriental and Australasian), separated by a transitional region (Wallacea) (Kingston 2010; Hall 2010). The main islands are Sumatra, Java, Borneo (partly, Kalimantan Province), Sulawesi and New Guinea (partly, provinces West Papua and Papua). Further there are large island groups like the Lesser Sunda Islands, the Moluccas and the Banda Islands.

Similarly, the Philippines is a complex archipelago with more than 7500 islands, spanning the AsianAustralian faunal zone interface directly at the Wallace Line. The Huxley's Line/Filter Zone divides the country into Palawan and associated islands, the presumed former land bridge to Northern Borneo, from the older land masses Mindoro, Romblon and Panay, and the truly oceanic islands of the Philippines. It has an extraordinary biodiversity, presumably supported by ancient land mass movements, environmental gradients along steep volcanic slopes and alterations of connectivity between neighboring islands due to sea-level regressions (Brown \& Diesmos 2010; Freitag et al. 2016).

Further collections of Ephemeroptera, especially of Baetidae, in Southeast Asia will unveil more unknown genera and many more species in the future.

\section{Material and methods}

The specimens from Sumatra and the Philippines were collected by Morgan Gueuning (University of Neuchâtel, Switzerland) and Jhoana Garces (Biodiversity Laboratory, Ateneo de Manila University, Philippines), respectively.

Specimens were preserved in 70-96\% ethanol. Larval dissection was carried out in Cellosolve (2-ethoxyethanol) with subsequent mounting on slides with Euparal liquid, using an Olympus SZX7 stereo microscope.

The DNA of part of the specimens was extracted using non-destructive methods, allowing subsequent morphological analysis (see Vuataz et al. 2011 for details). We amplified a 658 bp fragment of the mitochondrial gene cytochrome oxidase subunit 1 (COI) using the primers LCO 1490 (GGTCAACAAATCATAAAGATATTGG) and HCO 2198 (TAAACTTCAGGGTGACCAAAAAA- 
Table 1. Sequenced specimens of Procerobaetis gen. nov.

\begin{tabular}{ccccc}
\hline Species & Locality & $\begin{array}{c}\text { Specimen voucher } \\
\text { catatlogue \# }\end{array}$ & GenBank \# (COI) & $\begin{array}{c}\text { GenSeq } \\
\text { Nomenclature }\end{array}$ \\
\hline $\begin{array}{c}\text { P. leptobranchius } \\
\text { sp. nov. }\end{array}$ & Indonesia: Sumatra & GBIFCH00421984 & MN453816 & genseq-1 COI \\
& & GBIFCH00422019 & MN453817 & genseq-2 COI \\
$\begin{array}{c}\text { P. petersorum } \\
\text { sp. nov. }\end{array}$ & Indonesia: Sumatra & GBIFCH00421968 & MN453818 & genseq-2 COI \\
P. sp. C & Indonesia: Sumatra & GBIFCH00421974 & MN453815 & genseq-2 COI \\
genseq-4 COI \\
P. freitagi sp. nov. & Philippines: Mindoro & GBIFCH00515323 & MN453819 & genseq-4 COI \\
\hline
\end{tabular}

TCA) (Folmer et al. 1994). The PCR temperature progression was set as: $30 \mathrm{~s}$ at $98^{\circ} \mathrm{C} ; 10 \mathrm{~s}$ at $98^{\circ} \mathrm{C}$, $30 \mathrm{~s}$ at $50^{\circ} \mathrm{C}, 30 \mathrm{~s}$ at $72^{\circ} \mathrm{C}(\times 37$ cycles $) ; 120 \mathrm{~s}$ at $72^{\circ} \mathrm{C}$. Sequencing was done with Sanger's method (Sanger et al. 1977). The genetic variability between specimens was estimated using Kimura-2parameter distances (K2P, Kimura 1980), calculated with the program MEGA 7 (Kumar et al. 2016, http://www.megasoftware.net). The GenBank accession numbers are given in Table 1: nomenclature of gene sequences follows Chakrabarty et al. (2013).

Drawings were made using an Olympus BX43 microscope. Photographs of larvae were taken using a Canon EOS 6D camera and the Visionary Digital Passport imaging system (http://www.duninc.com) and processed with the programs Adobe Photoshop Lightroom (http://www.adobe.com) and Helicon Focus ver. 5.3 (http://www.heliconsoft.com). Photographs were subsequently enhanced with Adobe Photoshop Elements ver. 13.

The distribution maps were generated with the program SimpleMappr (https://simplemappr.net, Shorthouse 2010; Fig. 14). The GPS coordinates of the locations of examined specimens are given in Table 2 .

The terminology used follows Hubbard (1995) and Morihara \& McCafferty (1979).

\section{Abbreviations of depositories}

AdMU = Ateneo de Manila University, Quezon City (Philippines)

MZL = Museum of Zoology Lausanne (Switzerland)

PNM = Museum of Natural History of the Philippine National Museum, Manila (Philippines)

$\mathrm{ZSM}=$ Zoological States Collection Munich (Germany)

Table 2. GPS coordinates of the locations of examined specimens of Procerobaetis gen. nov.

\begin{tabular}{cccc}
\hline Species & Locality & \multicolumn{2}{c}{ GPS coordinates } \\
\hline P. leptobranchius sp. nov. & Indonesia: Sumatra & $00^{\circ} 23.54^{\prime} \mathrm{S}$ & $100^{\circ} 21.46^{\prime} \mathrm{E}$ \\
P. petersorum sp. nov. & & $00^{\circ} 23.06^{\prime} \mathrm{S}$ & $100^{\circ} 21.41^{\prime} \mathrm{E}$ \\
& Indonesia: Sumatra & $00^{\circ} 17.13^{\prime} \mathrm{S}$ & $100^{\circ} 41.23^{\prime} \mathrm{E}$ \\
& & $00^{\circ} 17.40^{\prime} \mathrm{S}$ & $100^{\circ} 40.84^{\prime} \mathrm{E}$ \\
& & $00^{\circ} 22.55^{\prime} \mathrm{S}$ & $100^{\circ} 39.55^{\prime} \mathrm{E}$ \\
$P$. sp. C & & $00^{\circ} 22.33^{\prime} \mathrm{S}$ & $100^{\circ} 41.75^{\prime} \mathrm{E}$ \\
$P$ freitagi sp. nov. & & $00^{\circ} 18.02^{\prime} \mathrm{S}$ & $100^{\circ} 40.13^{\prime} \mathrm{E}$ \\
& Indonesia: Sumatra & $00^{\circ} 23.65^{\prime} \mathrm{S}$ & $100^{\circ} 25.48^{\prime} \mathrm{E}$ \\
& Philippines: Luzon & $15^{\circ} 46.80^{\prime} \mathrm{N}$ & $121^{\circ} 13.28^{\prime} \mathrm{E}$ \\
\hline
\end{tabular}




\title{
Results
}

\author{
Class Insecta Linnaeus, 1758 \\ Order Ephemeroptera Hyatt \& Arms, 1891 \\ Family Baetidae Leach, 1815 \\ Procerobaetis Kaltenbach \& Gattolliat gen. nov. \\ urn:1sid:zoobank.org:act:AF35EE1B-253D-4A27-8B46-AB1BED25D1F9 \\ Figs $1-3,6 \mathrm{M}, 7 \mathrm{~A}, 12-14$
}

\section{Type species}

Procerobaetis leptobranchius gen. et sp. nov., by present designation.

\section{Diagnosis}

\section{Larva}

This new genus is distinguished by the combination of the following characters: A) body elongate and slender, abdomen dorsoventrally flattened, head and thorax in lateral view rounded, head clearly hypognathous (Figs 12-13); B) base of antennae close to each other, with carina between them (Fig. 6M); C) antenna without process on scape, with spines on apex of flagellum segments, maximally expressed on flagellum segment IX (Fig. 3A); D) labrum on dorsal surface with many long, stout, simple setae, erratically distributed, not arranged in one arc (Fig. 1A); E) right mandible with a row of thin setae at inner margin of innermost denticle, a simple stick-like, apicolaterally pectinate prostheca and a row of long, stout setae between prostheca and mola (Fig. 1B-C); F) left mandible with a row of short, stout setae and minute denticles between prostheca and mola, both directed toward subtriangular process, no setae at apex of mola (Fig. 1D); G) hypopharynx with a medial tuft of stout setae (Fig. 1F); H) 3-segmented maxillary palp much longer than galea-lacinia (Fig. 1G); I) glossae basally broad, narrowing toward apex, slightly shorter than paraglossae, ventral surface covered with erraticly distributed setae, paraglossae sub-rectangular, apically curved, labial palp segment II with small distolateral expansion, segment III sub-quadrangular (Fig. 1H); J) legs with short, laterally pectinate setae ventrally on femur, tibia and tarsus, femoral patch absent on all legs, patellotibial suture present on all legs, claw elongate and slender, pointed, slightly curved, with one row of many denticles increasing in length toward apex (Fig. 2A-F); K) gills elongate, distally noticeably pointed, at least gills I and II with very long, extended apex (Figs 3B-H, 10B-H); L) posterior margins of tergites I-VII without spines (Fig. 2G); M) paraproct without prolongation at posterior margin (Fig. 2H).

\section{Imagines}

Unknown.

\section{Etymology}

Procerobaetis is a combination of Procero - in reference to the elongate, slender habitus of the larvae - and baetis to highlight its superficial similarities with the genus Baetis Leach, $1815 \mathrm{~s}$. lat. The gender is masculine.

\section{Description: larva}

\section{Body}

Elongate and slender, abdomen dorsoventrally flattened, head and thorax in lateral view rounded, head hypognathous (Figs 12-13).

\section{Head}

AnTENNA (Figs 3A, 6M, 7A, 10A). Long, ca. $2 \times$ as long as head length; scape without process; scape and pedicel almost bare; flagellum with lanceolate spines at apex of each segment, longer at inner lateral 
margin, increasing in length distally until segment IX to XI and decreasing thereafter. Bases of antennae close to each other, with carina between them.

LABRUM (Fig. 1A). Rectangular, wider than long; dorsal surface with many long, stout, simple setae, erratically distributed and not arranged in an arc; distolateral margin with feathered setae, distomedial margin with bifid setae.

Right MANDiBLE (Fig.1B-C). Canine with largely fused incisors with well developed, apically rounded denticles, inner margin of innermost denticle with a row of thin setae; prostheca stick-like, apicolaterally pectinate; margin between prostheca and mola with a row of long, stout setae.

LeFT MANDiBLE (Fig. 1D-E). Canine with largely fused incisors with well developed, apically rounded denticles; prostheca robust, apically with small denticles and comb-shape structure; margin between prostheca and mola with a row of short stout setae and minute denticles, both directed toward subtriangular process; no setae at apex of mola.

HypopharynX (Fig. 1F). With a medial tuft of stout setae.

Maxilla (Fig. 1G). Apically with three stout teeth and three denti-setae, distal denti-seta tooth-like, other denti-setae slender, bifid and pectinate; maxillary palp with three segments, much longer than galea-lacinia.

Labium (Fig. 1H). Glossae basally broad, narrowing toward apex, slightly shorter than paraglossae, ventral surface covered with erratically distributed setae; paraglossae sub-rectangular, apically curved inward, apical margin with three rows of long setae; labial palp 3-segmented, segment II with small distolateral expansion, segment III sub-quadrangular. Mentum with scattered fine, simple setae at least in distal area.

\section{Thorax}

HindWING PADS (Fig. 2I). Absent.

Foreleg (Fig. 2A-F). Long and slender; dorsal margin of femur with a row of medium, spine-like setae and many stout, lanceolate, laterally pectinate setae scattered along ventral margin, femoral patch absent; dorsal margin of tibia bare or with a row of fine setae, ventral margin with a row of stout, lanceolate, laterally pectinate setae; tarsus dorsally bare or with a row of fine setae, ventral margin with a row of stout, lanceolate, laterally pectinate setae; tarsal claw elongate and slender, pointed, slightly curved, with one row of many denticles increasing in length toward apex, subapical setae absent.

\footnotetext{
Abdomen

TERgites (Fig. 2G). Posterior margin of segments I-VII smooth, without spines.

GiLls (Figs 3B-H, 10B-H). Seven pairs of single gills on segments I-VII, dorsally oriented, elongate, slender, distally noticeably pointed, at least gills I and II with very long, extended apex.

PARAPROCt (Fig. 2H). With stout marginal spines and without prolongation at posterior margin.

CAUDAL FILAMENTS (Fig. 13A). Inner margin of cerci with very thin, long setae; median caudal filament slightly shorter than cerci, bilaterally with very thin, long setae.
}

\section{Description: imagines}

Unknown. 


\section{Distribution (Fig. 14)}

Indonesia: Sumatra; Philippines: Luzon, Mindoro; Thailand (based on a photo provided by B. Boonsoong, pers. comm.). The Baetidae of Southeast Asia are still poorly known, and many locations have not yet been sampled at all. We expect a wider distribution and more species of this genus in Southeast Asia.

Procerobaetis leptobranchius Kaltenbach \& Gattolliat gen. et sp. nov. urn:lsid:zoobank.org:act:B05740B0-8BC1-4F5B-9F32-6852C54B9EB1

Figs $1-4,12 \mathrm{~A}-\mathrm{B}, 14 \mathrm{~B}$

\section{Diagnosis: larva}

The diagnostic characters of all species are summarized in Table 3.

\section{Etymology}

Procerobaetis leptobranchius gen. et sp. nov. is a combination of lepto - derived from the Greek word 'leptos', meaning 'fine', 'thin', 'slender' - and branchius, derived from the Latin word branchiae, meaning 'gills', in reference to the remarkably elongate and long-pointed gills I and II, which are typical for the genus. It is also in reference to Leptophlebiidae, where this type of gill partly exists as well.

\section{Material examined}

Holotype

INDONESIA • larva; Sumatra, volcano Singgalang, Caruak River; 00²3.06' S, $100^{\circ} 21.41^{\prime}$ E; $1640 \mathrm{~m}$ a.s.1.; 23 Mar. 2014; M. Gueuning leg.; on slide; voucher: GBIFCH 00421984; MZL.

\section{Paratypes}

INDONESIA - 4 larvae; same collection data as for holotype; on slides; vouchers: GBIFCH 00422019 , GBIFCH 00422023, GBIFCH 00421977, GBIFCH 00421989; MZL • 5 larvae; same collection data as for holotype; in alcohol; vouchers: GBIFCH 00421983, GBIFCH 00422974 (3 larvae), GBIFCH 00421986; MZL • 1 larva; Sumatra, volcano Singgalang, Pagu Pagu River; 00²3.54' S, $100^{\circ} 21.46^{\prime}$ E; 1785 m a.s.1.; 22 Mar. 2014; M. Gueuning leg.; on slide; voucher: GBIFCH 00421957; MZL.

Description: larva (Figs 1-4, 12A-B)

BODY LENGTH. 4.6-5.5 mm.

Colouration (Fig. 12A). Head, thorax and abdomen dorsally dark brown, head and thorax with bright median dorsal suture. Head and thorax ventrally brown, abdomen ventrally dark brown. Legs light brown, caudal filaments light brown without darker band or pattern.

\section{Head}

AnTENNA (Fig. 3A). Approximately $2 \times$ as long as head length; flagellum with lanceolate spines at apex of each segment, longer at inner lateral margin, increasing in length distally until segment IX and decreasing thereafter.

LABRUM (Fig. 1A). Rectangular, length $0.7 \times$ maximum width. Distal margin with medial emargination and a small process. Dorsally with long, fine, simple setae scattered over surface in proximal area; many long, stout, simple setae in anteromedial area, erratically distributed, not arranged in an arc. Ventrally with marginal row of setae composed of lateral and anterolateral long, feathered setae and medial long, bifid setae; ventral surface with about ten short, spine-like setae near lateral and anterolateral margins.

Right mandible (Fig. 1B-C). Outer and inner set of denticles with $4+3$ denticles. Margin between prostheca and mola straight, with a row of long, stout setae. Tuft of setae at apex of mola present. 


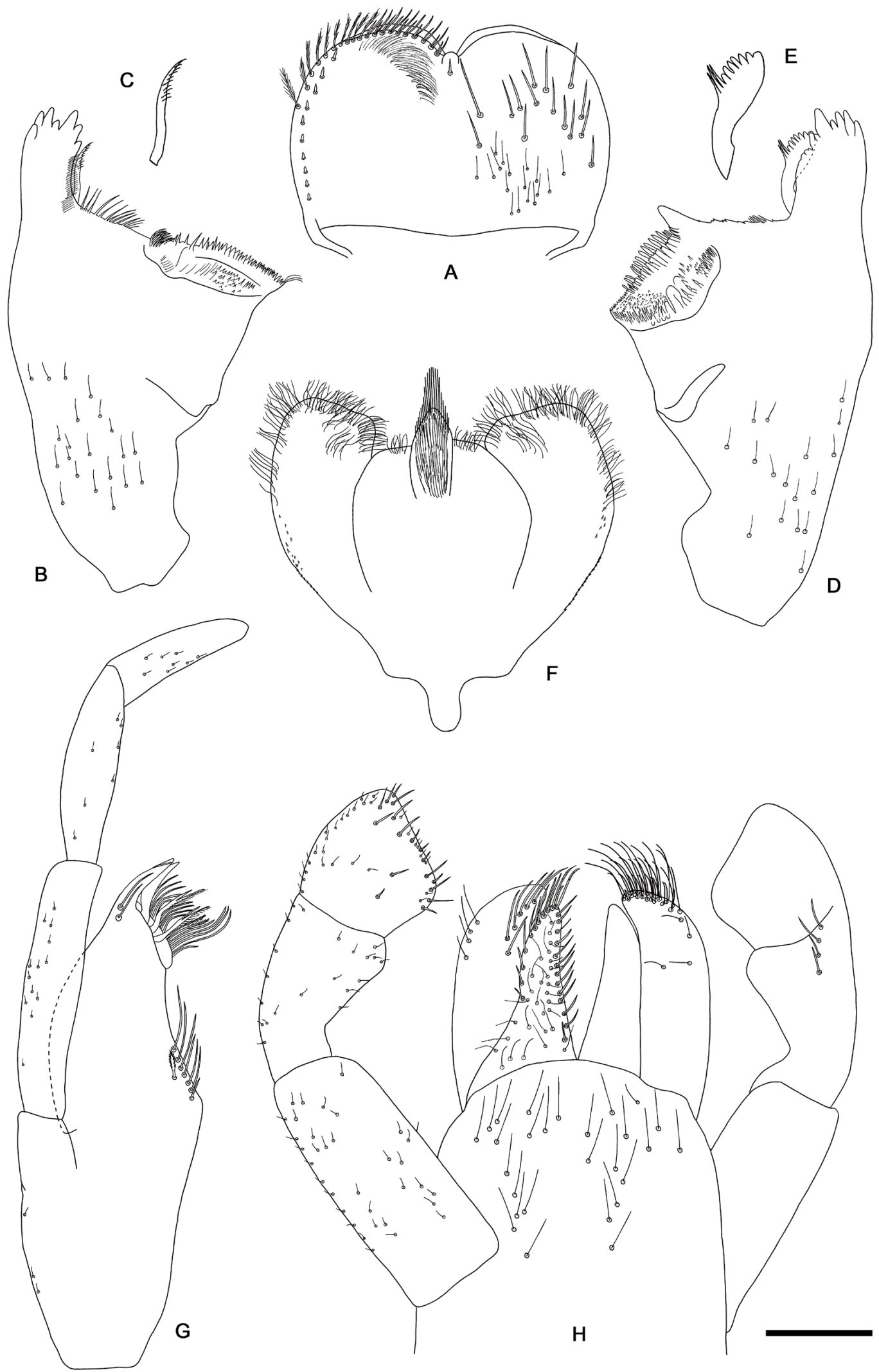

Fig. 1. Procerobaetis leptobranchius gen. et sp. nov., larval morphology. A. Labrum. B. Right mandible. C. Right prostheca. D. Left mandible. E. Left prostheca. F. Hypopharynx. G. Maxilla. H. Labium. Scale bar: $0.1 \mathrm{~mm}$. 
Left mandible (Fig. 1D-E). Outer and inner set of denticles with $4+3$ denticles. Subtriangular process long and slender, above level of area between prostheca and mola. Denticles of mola apically constricted. Setae at apex of mola absent. Both mandibles with lateral margins slightly convex. Basal half with fine, simple setae scattered over dorsal surface.

HypopharynX (Fig. 1F). Lingua shorter than superlingua, about as broad as long, with medial tuft of long, stout setae. Superlingua distally almost straight, lateral margin rounded, with fine, long, simple setae along distal and distolateral margin.

MaXILLA (Fig. 1G). Galea-lacinia with two simple, robust apical setae under crown. Medially with one pectinate, spine-like seta and a row of 6-8 medium to long, simple setae. Maxillary palp $2 \times$ as long as length of galea-lacinia; palp segment II $0.7 \times$ length of segment I, palp segment III $0.6 \times$ length of segment II; setae on maxillary palp fine, simple, scattered over surface of segments I, II and III; apex of last segment rounded.

LABIUM (Fig. 1H). Glossae basally broad, narrowing toward apex, slightly shorter than paraglossae; inner margin with 13-15 spine-like setae; apex with two long, robust, pectinate setae and one medium, robust, pectinate seta; outer margin with eight spine-like setae, increasing in length distally; ventral surface with medium, fine, simple, scattered setae. Paraglossae sub-rectangular, apically curved inward; apex rounded, with three rows of long, robust setae; ventrally four medium, simple setae in anteromedial area; dorsally with a row of 6-7 long, spine-like setae near inner margin and several medium, fine, simple setae at outer margin. Labial palp with segment I $0.9 \times$ length of segments II and III combined, ventrally with scattered short, fine, simple setae; segment II with small distolateral expansion, ventrally with scattered short, fine, simple setae, dorsally with a row of 4-5 long, spine-like setae; segment III subquadrangular, apex rounded, ventrally covered with short and medium spine-like, simple setae and short, fine, simple setae. Mentum medially and distally with scattered fine, simple setae.

\section{Thorax}

Foreleg (Figs 2A-F, 4A). Ratio of foreleg segments 1.3:1.0:0.9:0.3. Femur. Length 4.2-4.7× maximum width; dorsal margin with a row of 7-9 curved, spine-like setae; length of setae $0.3 \times$ maximum width of femur; apex rounded, with one pair of laterally slightly pectinate, spine-like setae; many stout, lanceolate, laterally pectinate setae scattered along ventral margin; femoral patch absent. Tibia. Dorsal margin bare; ventral margin with a row of curved, laterally pectinate, spine-like setae, on apex some longer, laterally pectinate, spine-like setae; anterior surface with scattered stout, lanceolate, laterally pectinate setae; patellotibial suture present on basal $1 / 3$. Tarsus. Dorsal margin bare; ventral margin with a row of curved, laterally pectinate, spine-like setae; tarsal claw elongate, slender, apically pointed, with one row of 7-8 larger denticles and many minute denticles, ventral margin at apex undulated, with many stripes.

MidDLE LEg (Fig. 4B). As foreleg, but dorsal margin of femur slightly concave, tibia at dorsal margin with a row of stout setae, and tarsal claw with one row of 5-11 larger denticles and many minute denticles.

HIND LEG (Fig. 4C). As foreleg, but dorsal margin of femur slightly concave, and tarsal claw with one row of 7-11 larger denticles and many minute denticles.

\footnotetext{
Abdomen

TERGITES (Fig. 2G). Surface with scattered scales and U-shaped scale bases. Posterior margin of tergites I-VIII without spines, tergite IX with triangular spines.

GILLS (Fig. 3B-H). Present on segments I-VII; elongate with pronounced points; gills I and II extremely slender and with very long, extended points; margin with very small denticles intercalating fine, simple setae; tracheae limited to main trunk. Gill I as long as length of segments II and $3 / 4$ of III combined, gill
} 


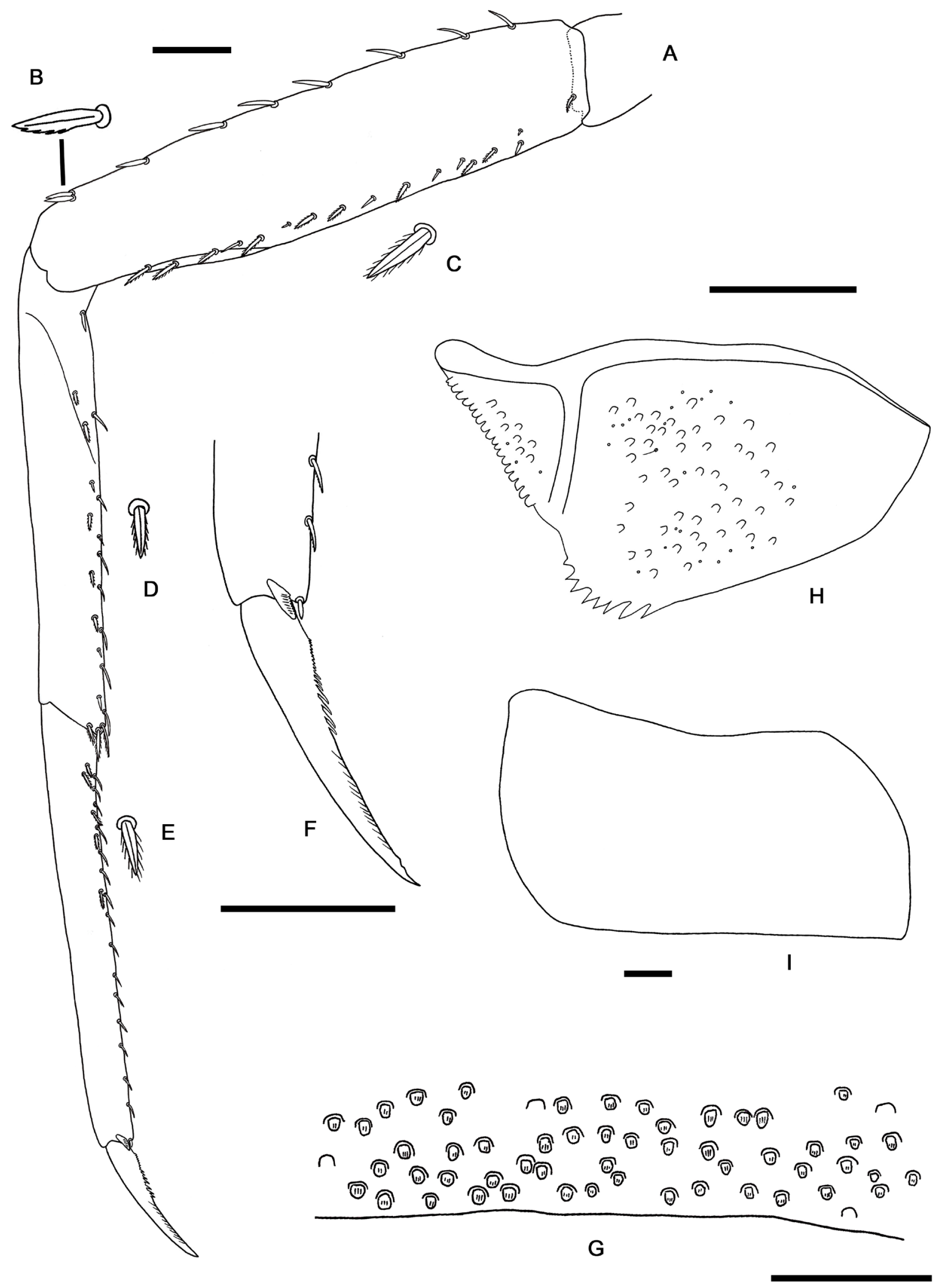

Fig. 2. Procerobaetis leptobranchius gen. et sp. nov., larval morphology. A. Foreleg. B. Seta at dorsal margin of femur (apex). C. Seta near ventral margin of femur. D. Seta at ventral margin and on ventral surface of tibia. E. Seta at ventral margin of tarsus. F. Fore claw. G. Tergum IV. H. Paraproct. I. Metanotum. Scale bars: $0.1 \mathrm{~mm}$. 

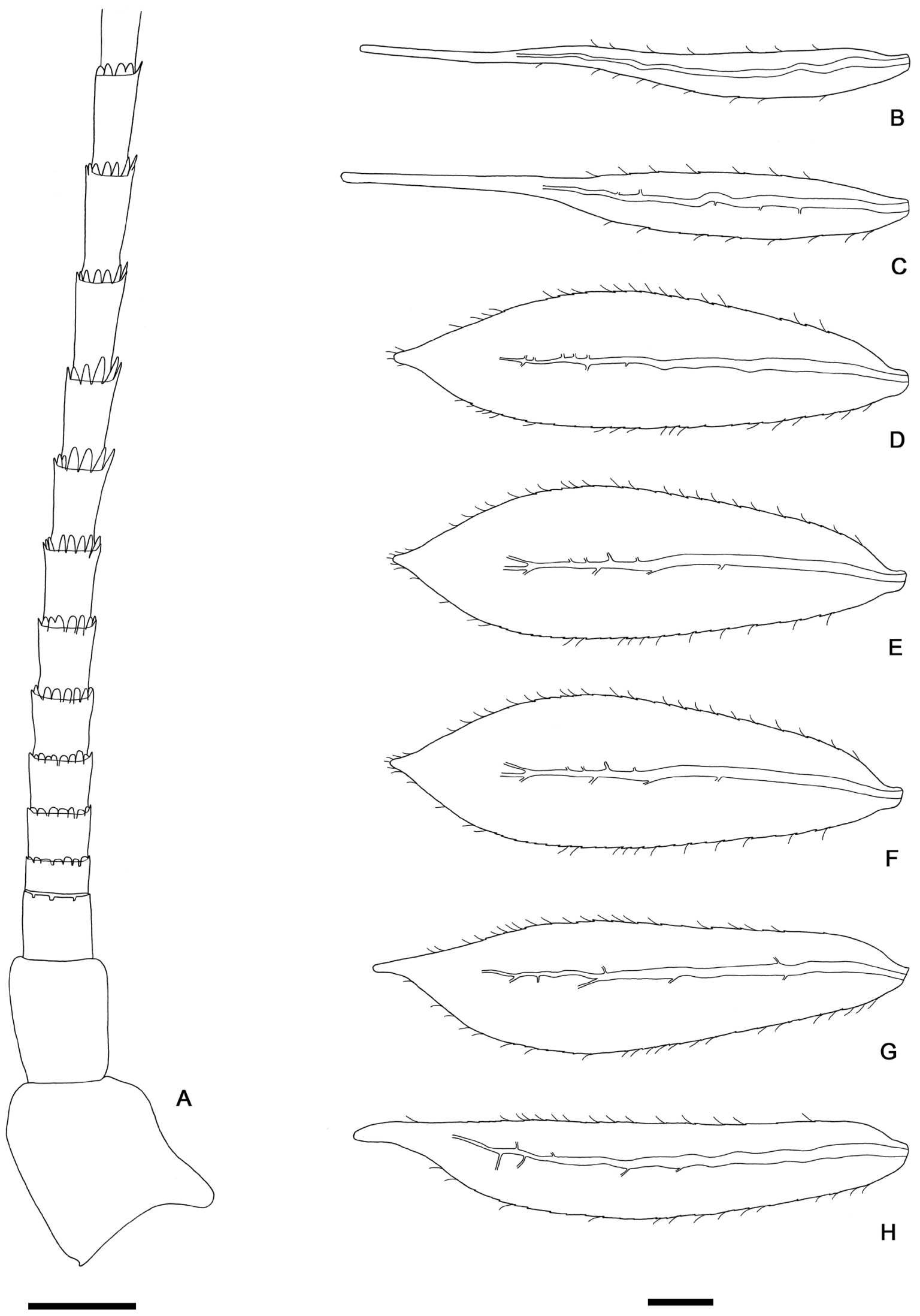

C
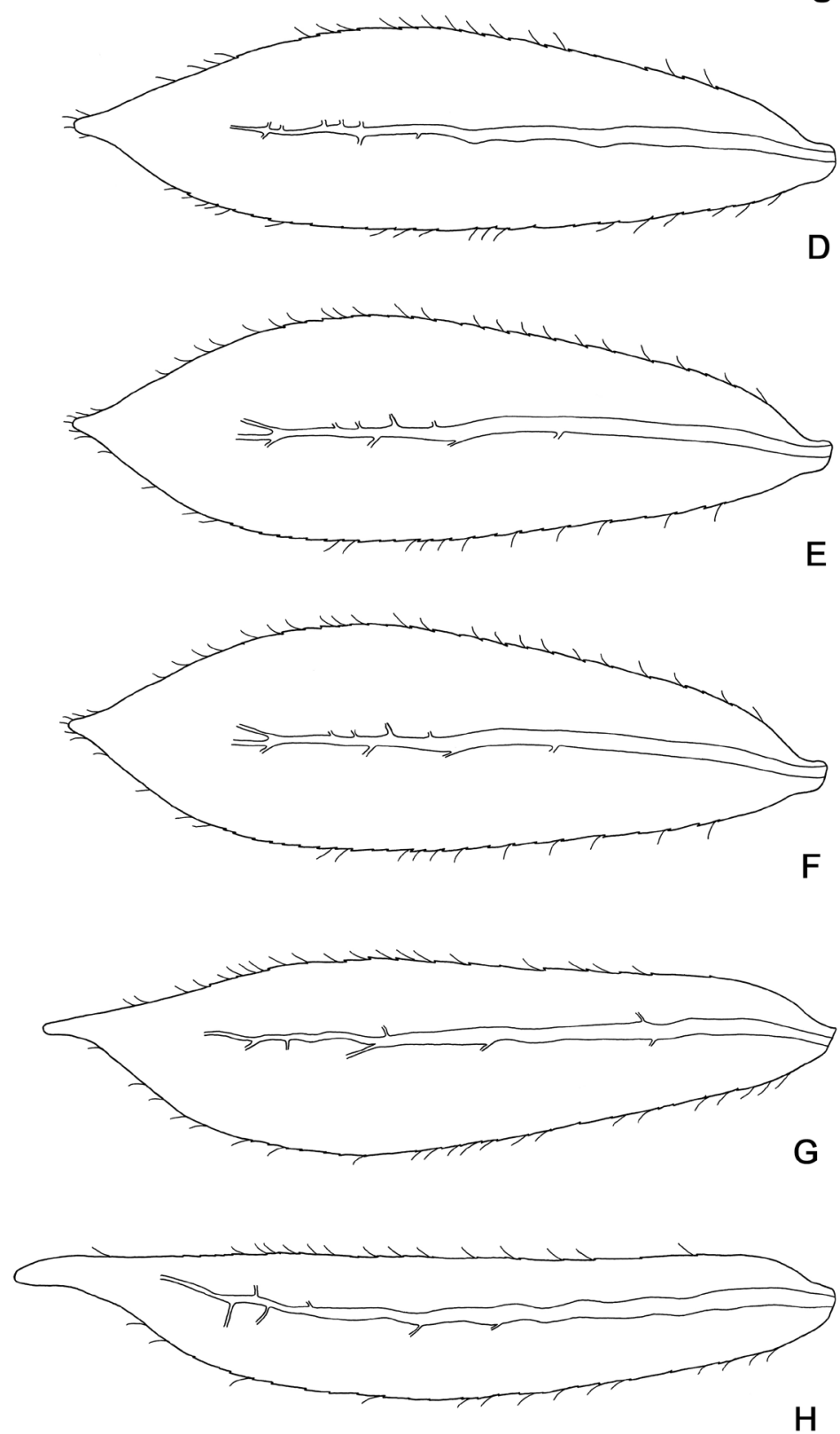

Fig. 3. Procerobaetis leptobranchius gen. et sp. nov., larval morphology. A. Antenna. B. Gill I. C. Gill II. D. Gill III. E. Gill IV. F. Gill V. G. Gill VI. H. Gill VII. Scale bars: 0.1 mm. 
II as long as length of segments III and IV combined, gill III as long as length of segments IV and $3 / 4$ of V combined, gill IV as long as length of segments V and VI combined, gill V as long as length of segments VI and VII combined, gill VI as long as length of segments VII to $1 / 2$ of IX combined, gill VII as long as length of segments VIII to X combined.

PARAPRoct (Fig. 2H). Posterior margin with 5-9 stout spines; surface with scattered U-shaped scale bases, micropores and a few fine, simple setae; posterolateral extension (cercotractor) with numerous small, marginal spines.

Caudal Filaments (Fig. 12A). Cerci ca $0.4 \times$ body length.

\section{Distribution}

Indonesia: Sumatra (Fig. 14B).

\section{Remarks}

The specimens were collected at altitudes from 1600 to $1800 \mathrm{~m}$ a.s.l. in two small, shallow, slow and rather cool forest streams (width ca $1 \mathrm{~m}$, depth $5-30 \mathrm{~cm}$, velocity $0.15-0.2 \mathrm{~m} / \mathrm{s}$, temperature $15.5-17^{\circ} \mathrm{C}$,
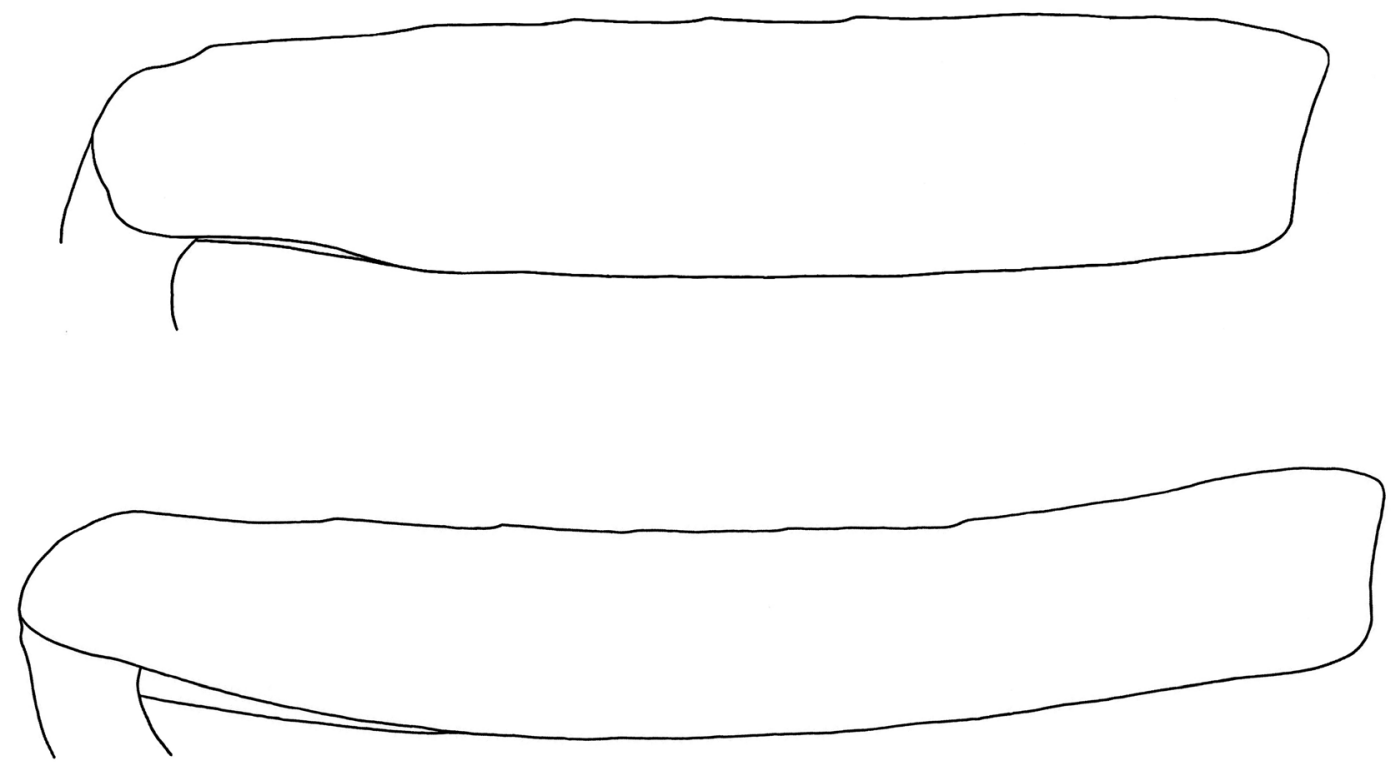

B

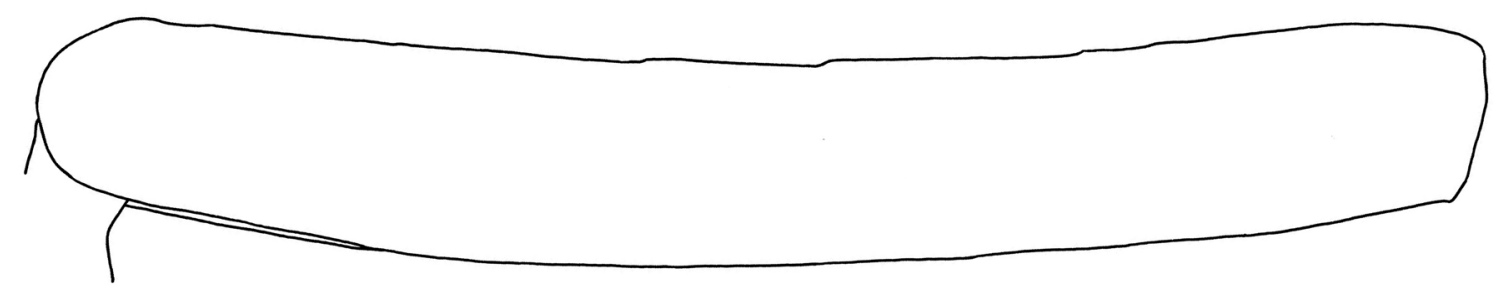

C

Fig. 4. Procerobaetis leptobranchius gen. et sp. nov., larval morphology. A. Foreleg femur. B. Middle leg femur. C. Hind leg femur. Scale bar: $0.1 \mathrm{~mm}$. 
pH 6-7) with partly to completely closed canopy. The substrate in both streams was dominated by boulders, stones and gravel with some sandy patches and with or without patches of leaf litter or dead wood.

Procerobaetis petersorum Kaltenbach \& Gattolliat gen. et sp. nov. urn:1sid:zoobank.org:act:8E7DA59C-2AB6-4518-BE84-5C71C5A5BD03

Figs 5-7, 12C-D, 14B

\section{Diagnosis: larva}

The diagnostic characters of all species are summarized in Table 3.

\section{Etymology}

P. petersorum gen. et sp. nov. is dedicated to the well-known mayfly researchers Janice Peters and her late husband William L. Peters for their contribution to our knowledge of Leptophlebiidae in Southeast Asia. Specific reference is given to the remarkable gills of Procerobaetis gen. nov., which are similar to those of some species of Leptophlebiidae.

\section{Material examined}

\section{Holotype}

INDONESIA • larva; Sumatra, volcano Sago, Kobun River; 00²2.55' S, 100³9.55' E; 1095 m a.s.l.; 19 Mar. 2014; M. Gueuning leg.; on slide; voucher no.: GBIFCH 00421980; MZL.

\section{Paratypes}

INDONESIA - 1 larva; same collection data as for holotype; on slide; voucher: GBIFCH 00421975 ; MZL • 3 larvae; same collection data as for holotype; in alcohol; vouchers: GBIFCH 00422930, GBIFCH 00422044, GBIFCH 00422751; MZL • 1 larva; Sumatra, volcano Sago, Simbukan River, 00 $17.40^{\prime}$ S, $100^{\circ} 40.84^{\prime}$ E; 955 m a.s.1.; 18 Mar. 2014; M. Gueuning leg.; on slide; voucher: GBIFCH 00422039;

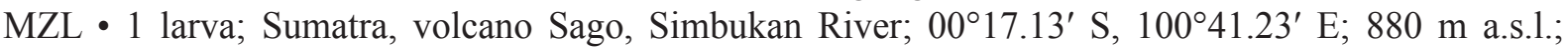
18 Mar. 2014; M. Gueuning leg.; in alcohol; voucher: GBIFCH 00422000; MZL • 1 larva; Sumatra, volcano Sago, Tampo River; $00^{\circ} 22.33^{\prime}$ S, $100^{\circ} 39.55^{\prime}$ E; 960 m a.s.1.; 20 Mar. 2014; M. Gueuning leg.; on slide; voucher: GBIFCH 00421968; MZL • 4 larvae; same collection data as for preceding; in alcohol; vouchers: GBIFCH 00422045, GBIFCH 00421964, GBIFCH 00422002, GBIFCH 00422705; MZL • 2 larvae; Sumatra, volcano Sago, Kaligain River; $00^{\circ} 18.02^{\prime}$ S, $100^{\circ} 40.13^{\prime}$ E; 1040 m a.s.l.; 5 Apr. 2014; M. Gueuning leg.; on slides; vouchers: GBIFCH 00421988, GBIFCH 00422006; MZL • 3 larvae; same collection data as for preceding; in alcohol; vouchers: GBIFCH 00422613, GBIFCH 00422043, GBIFCH 00422003; MZL.

Description: larva (Figs 5-7, 12C-D)

Body LeNGTH. 3.4-4.5 mm.

Colouration (Fig. 12B). Head, thorax and abdomen dorsally brown, head and thorax with bright median dorsal suture. Head and thorax ventrally light brown, abdomen ventrally brown. Legs light brown, caudal filaments light brown without darker band or pattern.

\section{Head}

AntenNa (Fig. 7A). Flagellum with lanceolate spines at apex of each segment, longer at inner lateral margin, increasing in length distally until segment IX and decreasing thereafter.

LABRUM (Fig. 5A). Rectangular, length $0.6 \times$ maximum width. Distal margin with medial emargination and a small process. Dorsally with long, fine, simple setae scattered over surface in proximal area; many 
long, stout, simple setae in anteromedial area, erratically distributed, not arranged in an arc. Ventrally with marginal row of setae composed of lateral and anterolateral long, feathered setae and medial long, bifid, pectinate setae; ventral surface with about five short, spine-like setae near lateral and anterolateral margin.

Right MAndible (Fig. 5B-C). Outer and inner set of denticles with $4+3$ denticles. Margin between prostheca and mola straight, with a row of long, stout setae. Tuft of setae at apex of mola present.

LeFt MANDiBle (Fig. 5D-E). Outer and inner set of denticles with $4+3$ denticles. Subtriangular process long and slender, above level of area between prostheca and mola. Denticles of mola apically constricted. Setae at apex of mola absent. Both mandibles with lateral margins almost straight. Basal half with fine, simple setae scattered over dorsal surface.

HypophaRYNX (Fig. 5F). Lingua shorter than superlingua, longer than broad, with medial tuft of long, stout setae. Superlingua distally almost straight, lateral margin rounded, with fine, long, simple setae along distal margin.

MAXILLA (Fig. 5G). Galea-lacinia with two simple, robust apical setae under crown. Medially with one pectinate, spine-like seta and a row of 5-6 medium to long, simple setae. Maxillary palp $2 \times$ as long as length of galea-lacinia; palp segment II $0.7 \times$ length of segment I, palp segment III $0.7 \times$ length of segment II; setae on maxillary palp fine, simple, scattered over surface of segments I, II and III; apex of last segment rounded.

LABIUM (Fig. 5H). Glossae basally broad, narrowing toward apex, slightly shorter than paraglossae; inner margin with 9-10 spine-like setae; apex with two long and one medium-length, robust, pectinate setae; outer margin with 5-7 spine-like setae, increasing in length distally; ventral surface with medium, fine, simple, scattered setae. Paraglossae sub-rectangular, apically curved inward; apex rounded, with three rows of long, robust setae; ventrally 1-3 medium, simple setae in anteromedial area; dorsally with a row of 5 long, spine-like setae near inner margin and several medium, fine, simple setae at outer margin. Labial palp with segment I $0.6 \times$ length of segments II and III combined, ventrally with scattered short, fine, simple setae; segment II with small distolateral expansion, ventrally with scattered short, fine, simple setae, dorsally with a row of 3-4 long, spine-like setae; segment III subquadrangular, apex rounded, ventrally covered with short, spine-like, simple setae and short, fine, simple setae. Mentum distally with scattered fine, simple setae.

\section{Thorax}

ForeLEg (Figs 6A-H, 7B). Ratio of foreleg segments 1.4:1.0:1.0:0.3. Femur. Length 3.1-3.8 $\times$ maximum width; dorsal margin with a row of 7-9 curved, spine-like setae; length of setae $0.26 \times$ maximum width of femur; apex rounded, with one pair of laterally slightly pectinate, spine-like setae; many stout, lanceolate, laterally pectinate setae scattered along ventral margin; femoral patch absent. Tibia. Dorsal margin bare; ventral margin with a row of curved, laterally pectinate, spine-like setae, at apex some longer, laterally pectinate, spine-like setae; anterior surface with scattered stout, lanceolate, laterally pectinate setae; patellotibial suture present on basal $1 \frac{1}{3}$. Tarsus. Dorsal margin bare; ventral margin with a row of curved, laterally pectinate, spine-like setae; tarsal claw elongate, slender, apically pointed, with one row of 4-7 larger denticles and many minute denticles, ventral margin at apex undulated, with many stripes.

MidDLE Leg (Fig. 7C). As foreleg, but dorsal margin of femur almost straight, tibia at dorsal margin with a row of stout setae, and tarsal claw with one row of 3-6 larger denticles and many minute denticles.

HIND LEG (Fig. 7D). As foreleg, but dorsal margin of femur almost straight, tarsal claw with one row of 4-7 larger denticles and many minute denticles. 


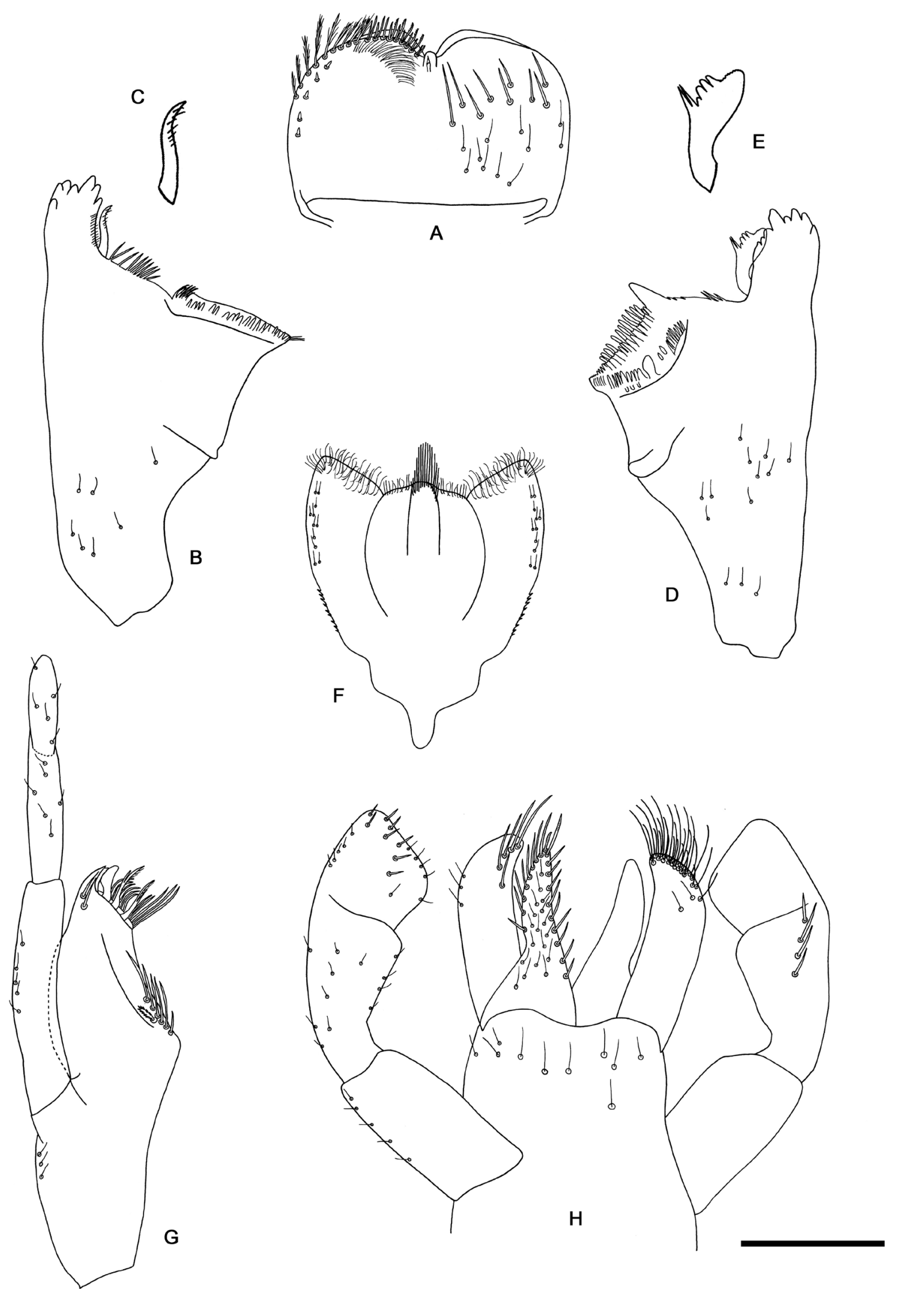

Fig. 5. Procerobaetis petersorum gen. et sp. nov., larval morphology. A. Labrum. B. Right mandible. C. Right prostheca. D. Left mandible. E. Left prostheca. F. Hypopharynx. G. Maxilla. H. Labium. Scale bar: $0.1 \mathrm{~mm}$. 


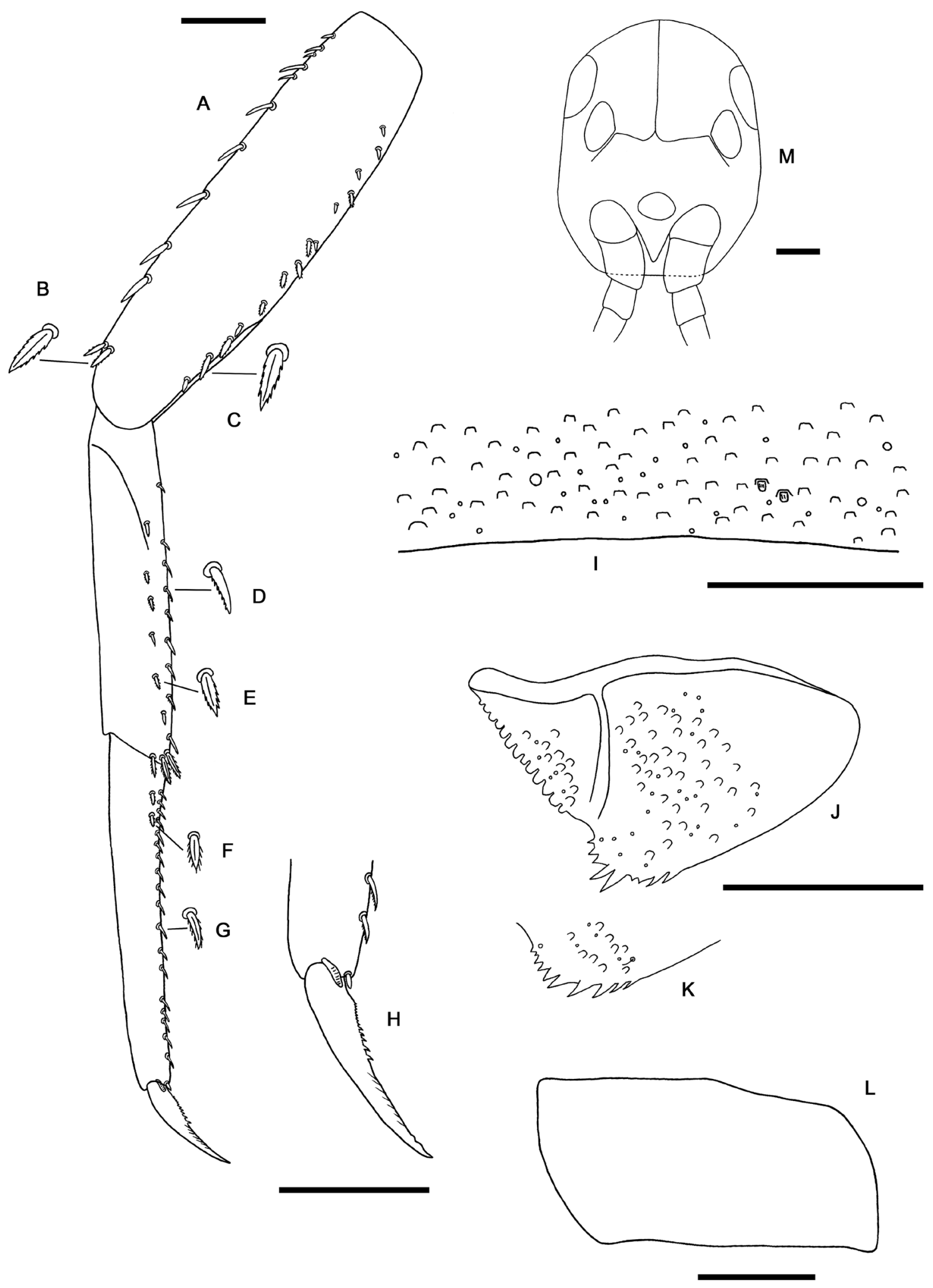

Fig. 6. Procerobaetis petersorum gen. et sp. nov., larval morphology. A. Foreleg. B. Seta on dorsal margin of femur (apex). C. Seta on ventral margin of femur. D. Seta on ventral margin of tibia. E. Seta on ventral surface of tibia. F. Seta near ventral margin of tarsus. G. Seta on ventral margin of tarsus. H. Fore claw. I. Tergum IV. J. Paraproct. K. Paraproct, posterior margin. L. Metanotum. M. Head. Scale bars: $0.1 \mathrm{~mm}$. 


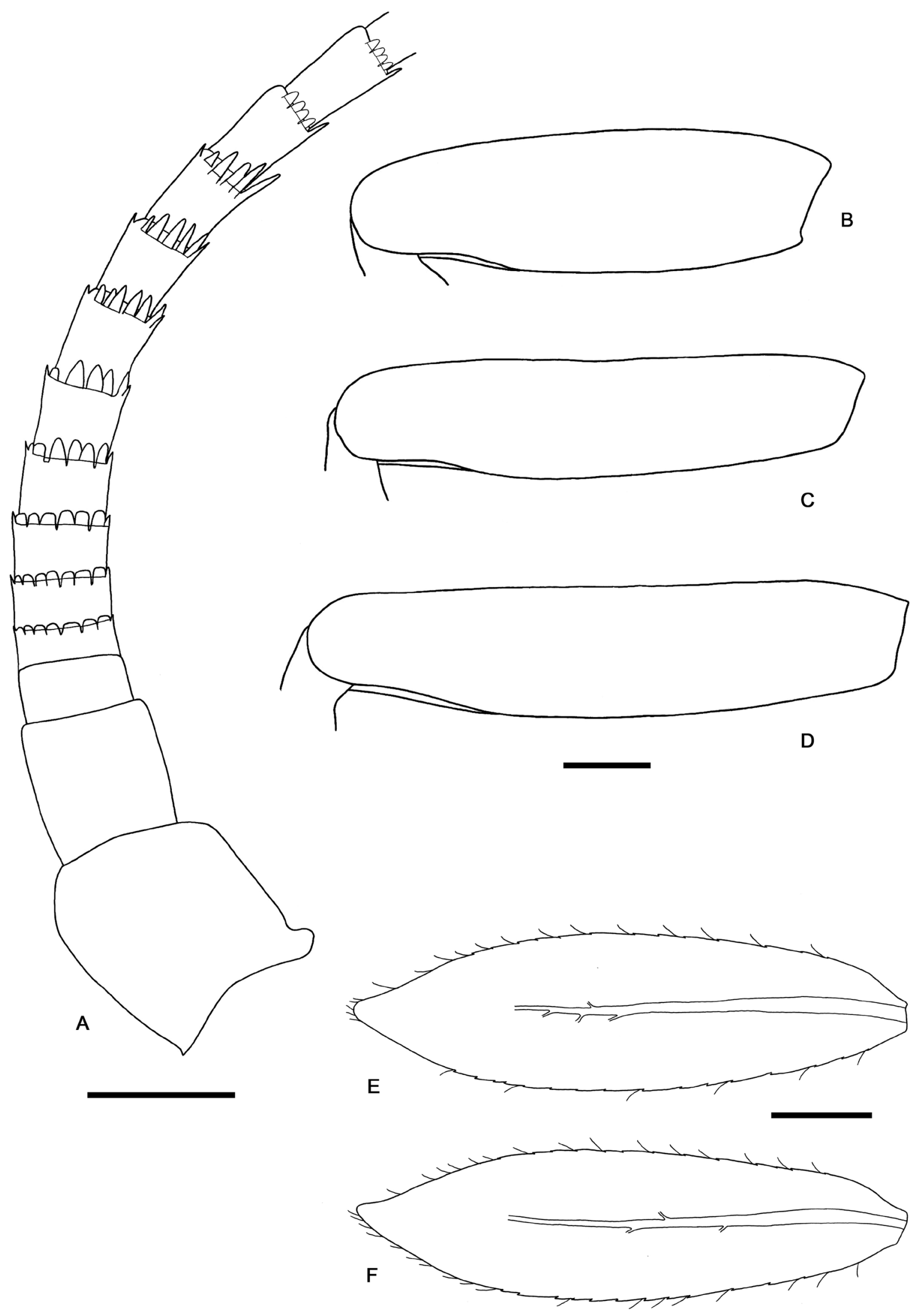

Fig. 7. Procerobaetis petersorum gen. et sp. nov., larval morphology. A. Antenna. B. Fore femur. C. Middle femur. D. Hind femur. E. Gill IV. F. Gill V. Scale bars: $0.1 \mathrm{~mm}$. 


\begin{abstract}
Abdomen
Tergites (Fig. 6I). Surface with scattered scales, U-shaped scale bases and micropores. Posterior margin of tergites I-VIII without spines, tergite IX with triangular spines.

GILLs (Fig. 7E-F). Present on segments I-VII; elongate, with pronounced points; gills I and II extremely slender and with very long, extended points; margin with very small denticles intercalating fine, simple setae; tracheae limited to main trunk. Gill II as long as length of segments III and $1 / 2$ of IV combined, gill III as long as length of segments IV and $1 / 2$ of V combined, gill IV as long as length of segments $\mathrm{V}$ and $1 / 2$ of VI combined, gill V as long as length of segments VI and $2 / 3$ of VII combined, gill VI as long as length of segments VII and VIII combined, gill VII as long as length of segments VIII and $2 / 3$ of IX combined.
\end{abstract}

Paraproct (Fig. 6J-K). Posterior margin with 8-10 stout spines; surface with scattered U-shaped scale bases and micropores; posterolateral extension (cercotractor) with numerous small, marginal spines.

Caudal filaments (Fig. 12C). Cerci ca $0.5 \times$ body length.

\title{
Distribution
}

Indonesia: Sumatra (Fig. 14B).

\section{Remarks}

The specimens were collected at altitudes from 800 to $1100 \mathrm{~m}$ a.s.1. in medium to large, mainly shallow and slow to moderate fast streams (width $2-10 \mathrm{~m}$, depth $20-150 \mathrm{~cm}$, velocity $0.28-0.5 \mathrm{~m} / \mathrm{s}$, temperature $\left.20-21^{\circ} \mathrm{C}, \mathrm{pH} 7-7.5\right)$. The streams were mostly in forests with a partly closed canopy, the substrate was dominated by bedrock, boulders and stones with few patches of leaf litter or dead wood. One stream was in a more disturbed area (rice fields, waste and soap).

Procerobaetis freitagi Kaltenbach \& Gattolliat gen. et sp. nov. urn:1sid:zoobank.org:act:8F16E037-FA56-46C9-BE5F-1530CB545115

Figs $8-11,13,14 \mathrm{C}$

\section{Diagnosis: larva}

The main diagnostic character is the shape of gills III-VII, with long extended points at the apex (Fig. 10D-H). The diagnostic characters of all species are summarized in Table 3.

\section{Etymology}

P. freitagi gen. et sp. nov. is dedicated to Professor Hendrik Freitag (Ateneo de Manila University) for his outstanding contribution to aquatic insect studies and for leading the passionate group of aquatic entomologists in the Philippines.

\section{Material examined}

Holotype

PHILIPPINES - larva; Luzon, Nueva Ecija, Pantabangan, Candaclan River, rural; $15^{\circ} 46.80^{\prime} \mathrm{N}$, 121⒔28' E; 240 m a.s.1.; 5 Feb. 1998; Mendoza leg.; on slide; voucher: GBIFCH 00592245; PNM.

\section{Paratypes}

PHILIPPINES • 1 larva; same collection data as for holotype; on slide; voucher GBIFCH 00515324; ZSM 1 larva; same collection data as for holotype; on slide; voucher: GBIFCH 00658095; AdMU • 1 larva; same collection data as for holotype; in alcohol; voucher: GBIFCH 00515331; ZSM. 


\section{Other material}

PHILIPPINES - 1 larva; Oriental Mindoro, Roxas, Barangay San Vicente, lower reach of Taugad River; $12^{\circ} 37.30^{\prime} \mathrm{N}, 121^{\circ} 22.97^{\prime}$ E; 140 m a.s.l.; 8 Nov. 2018; J. Garces leg.; on slide; voucher: GBIFCH 00515323; ZSM.

Description: larva (Figs 8-11, 13A-B)

Body LeNGTH. 4.5-4.8 mm.

Colouration (Fig. 13A). Head, thorax and abdomen dorsally brown, head and thorax with bright median dorsal suture. Head, thorax and abdomen ventrally light brown, abdominal segments VI to IX gradually darker. Legs transparent, caudal filaments transparent.

\section{Head}

ANTENNA (Fig. 10A). Approximately $2 \times$ as long as head length; flagellum with lanceolate spines at apex of each segment, longer at inner lateral margin, increasing in length distally until segment IX-XI and decreasing thereafter.

LABRUM (Fig. 8A). Rectangular, length $0.6 \times$ maximum width. Distal margin with medial emargination and a small process. Dorsally with some medium, fine, simple setae and some medium, stout, simple setae scattered over surface in proximal area; many long, stout, simple setae in anteromedial area, erratically distributed, not arranged in an arc. Ventrally with marginal row of setae composed of lateral and anterolateral long, feathered setae and medial long, bifid, pectinate setae; ventral surface with about five short, spine-like setae near lateral and anterolateral margin.

Right Mandible (Fig. 8B-C). Outer and inner set of denticles with $4+1+3$ denticles. Margin between prostheca and mola straight, with a row of long, stout setae. Tuft of setae at apex of mola present.

LefT MANDible (Fig. 8D-E). Outer and inner set of denticles with $4+3$ denticles. Subtriangular process long and slender, above level of area between prostheca and mola. Denticles of mola apically constricted. Setae at apex of mola absent. Both mandibles with lateral margins almost straight. Basal half with fine, simple setae scattered over dorsal surface.

HypopharynX (Fig. 8F). Lingua shorter than superlingua, longer than broad, with medial tuft of long, stout setae. Superlingua distally almost straight, lateral margin rounded, with fine, long, simple setae along distal margin.

Maxilla (Fig. 8g). Galea-lacinia with two simple, robust apical setae under crown. Medially with one pectinate, spine-like seta and a row of 5-6 long, simple setae. Maxillary palp $2 \times$ as long as length of galea-lacinia; palp segment II $0.7 \times$ length of segment I, palp segment III $0.5 \times$ length of segment II; setae on maxillary palp fine, simple, scattered over surface of segments I, II and III; apex of last segment rounded.

LABIUM (Fig. 8H). Glossae basally broad, narrowing toward apex, slightly shorter than paraglossae; inner margin with 9-10 spine-like setae; apex with two long and one medium, robust, pectinate setae; outer margin with seven spine-like setae, increasing in length distally; ventral surface with medium, fine, simple, scattered setae. Paraglossae sub-rectangular, apically curved inward; apex rounded, with three rows of long, robust setae; ventrally two medium, simple setae in anteromedial area; dorsally with a row of five long, spine-like setae near inner margin. Labial palp with segment I $0.9 \times$ length of segments II and III combined, ventrally with scattered short, fine, simple setae; segment II with very small distomedial expansion, ventrally with scattered short, fine, simple setae, dorsally with a row of 


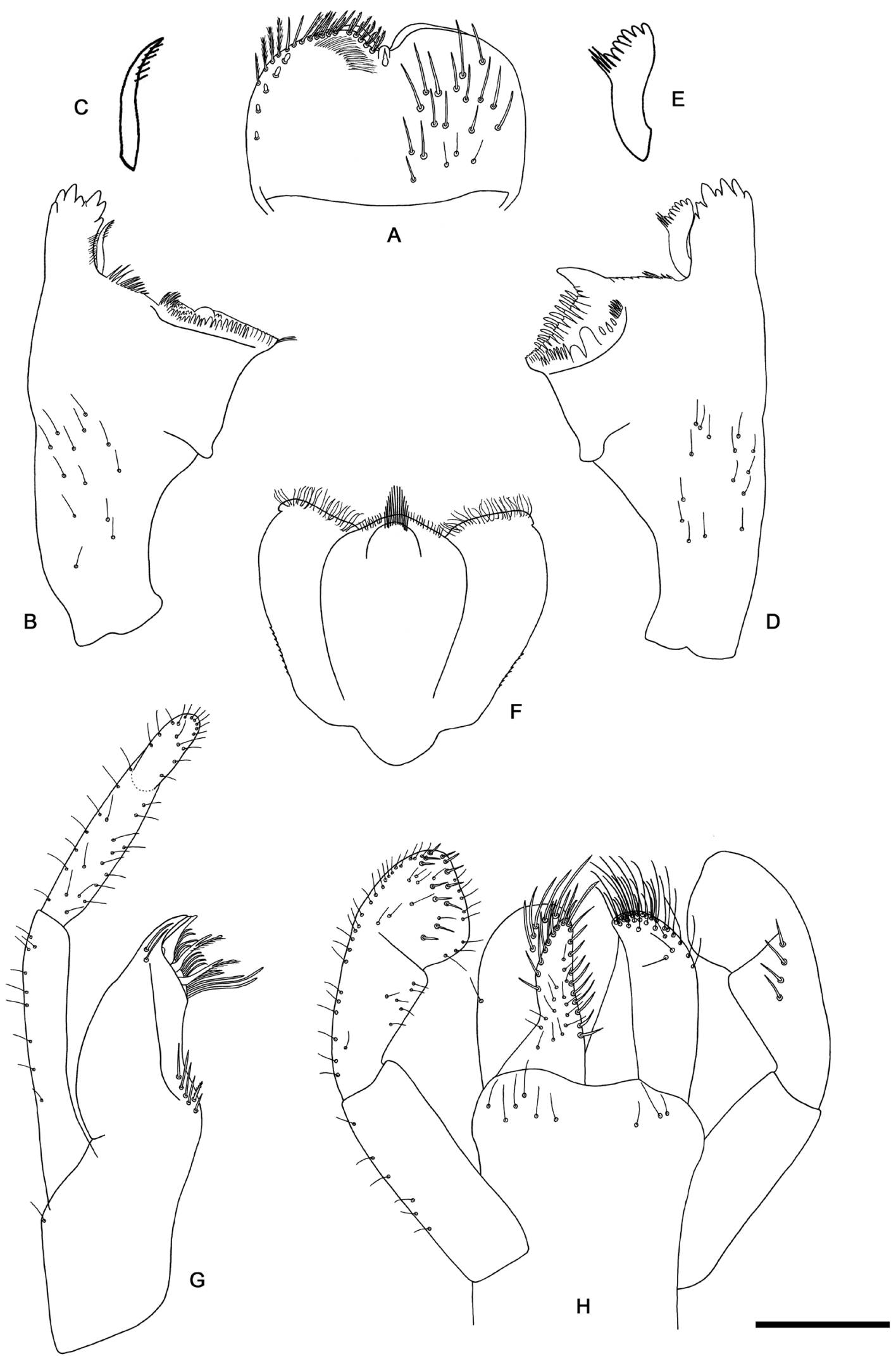

Fig. 8. Procerobaetis freitagi gen. et sp. nov., larval morphology. A. Labrum. B. Right mandible. C. Right prostheca. D. Left mandible. E. Left prostheca. F. Hypopharynx. G. Maxilla. H. Labium. Scale bar: $0.1 \mathrm{~mm}$. 


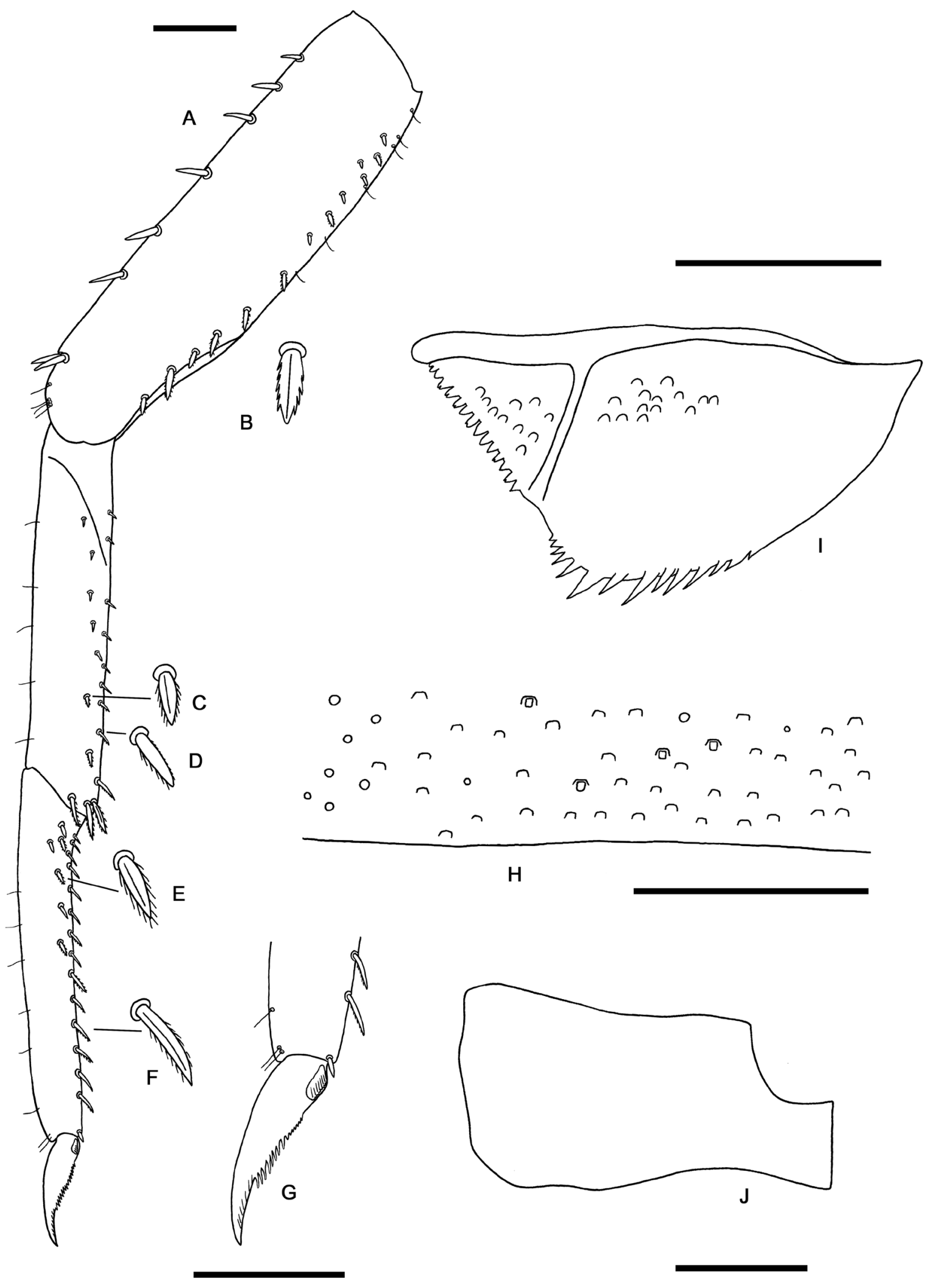

Fig. 9. Procerobaetis freitagi gen. et sp. nov., larval morphology. A. Foreleg. B. Seta on ventral margin of femur. C. Seta on ventral surface of tibia. D. Seta on ventral margin of tibia. E. Seta on ventral surface of tarsus. F. Seta on ventral margin of tarsus. G. Fore claw. H. Tergum IV. I. Paraproct. J. Metanotum. Scale bars: $0.1 \mathrm{~mm}$. 

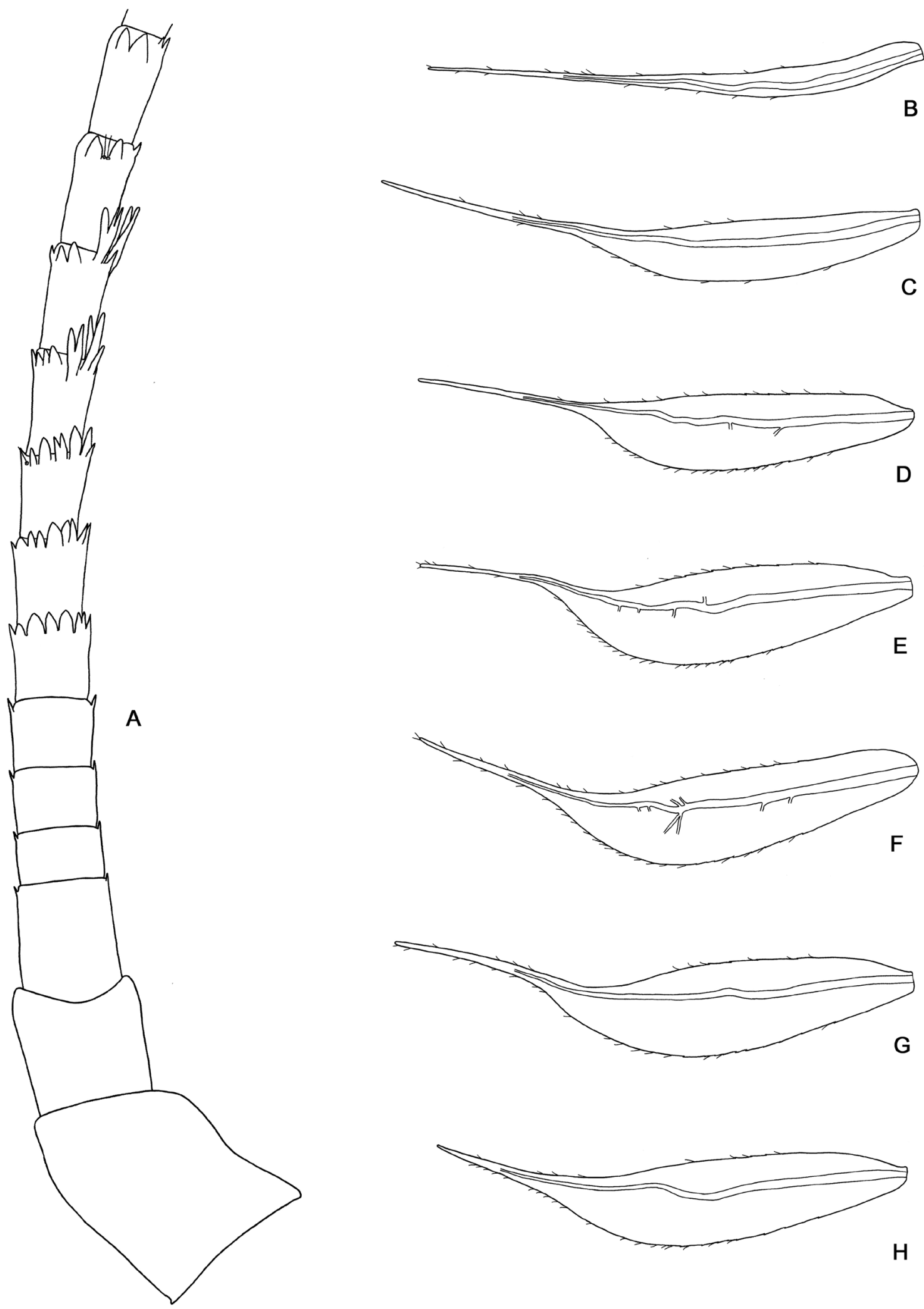

Fig. 10. Procerobaetis freitagi gen. et sp. nov., larval morphology. A. Antenna. B. Gill I. C. Gill II. D. Gill III. E. Gill IV. F. Gill V. G. Gill VI. H. Gill VII. Scale bars: $0.1 \mathrm{~mm}$. 
4-6 long, spine-like setae; segment III subquadrangular, apex rounded, ventrally covered with short spine-like, simple setae and short, fine, simple setae. Mentum distally with scattered fine, simple setae.

\section{Thorax}

Foreleg (Figs 9A-G, 11A). Ratio of foreleg segments 1.4:1.0:1.0:0.3. Femur. Length 3.5-3.8× maximum width; dorsal margin with a row of 6-7 curved, spine-like setae; length of setae $0.25 \times$ maximum width of femur; apex rounded, with one pair of spine-like setae; many stout, lanceolate, laterally pectinate setae scattered along ventral margin; femoral patch absent. Tibia. Dorsal margin with a row of fine, simple setae; ventral margin with a row of curved, laterally pectinate, spine-like setae, at apex some longer, laterally pectinate, spine-like setae; anterior surface with scattered stout, lanceolate, laterally pectinate setae; patellotibial suture present on basal $1 / 3$. Tarsus. Dorsal margin with a row of

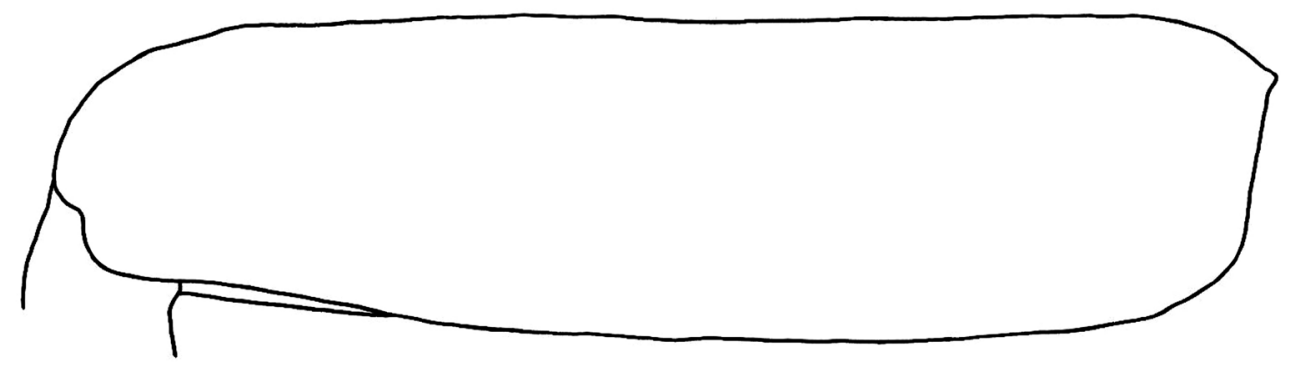

A

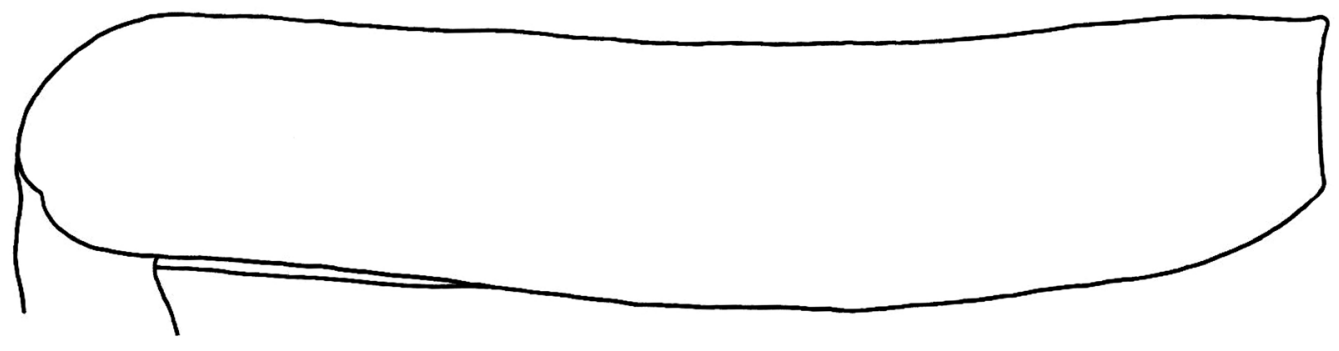

B

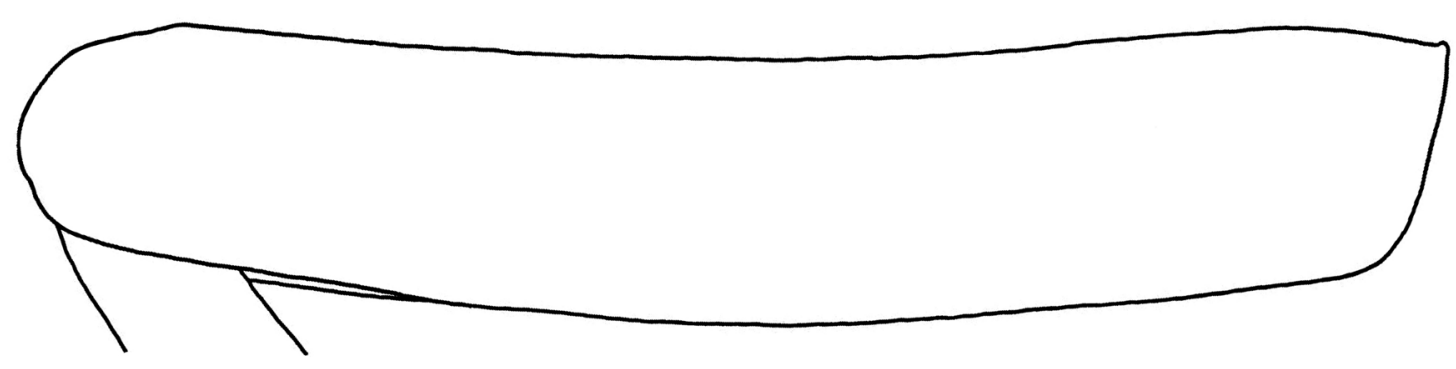

C

Fig. 11. Procerobaetis freitagi gen. et sp. nov., larval morphology. A. Fore femur. B. Middle femur. C. Hind femur. Scale bar: $0.1 \mathrm{~mm}$. 
fine, simple setae; ventral margin with a row of curved, laterally pectinate, spine-like setae; tarsal claw elongate, slender, apically pointed, with one row of 7-10 larger denticles and many minute denticles, ventral margin at apex straight, with many stripes.

MidDLE LEg (Fig. 11B). As foreleg, but dorsal margin of femur slightly concave.

HIND LEG (Fig. 11C). As foreleg, but dorsal margin of femur slightly concave and tarsal claw with one row of 10-12 larger denticles and many minute denticles.

\begin{abstract}
Abdomen
Tergites (Fig. 9H). Surface with scattered scales, U-shaped scale bases and micropores. Posterior margin of tergites VIII and IX with triangular spines.

GILLS (Fig. 10B-H). Present on segments I-VII; elongate, with very long, extended points; margin with very small denticles intercalating fine, simple setae; tracheae limited to main trunk. Gill I as long as length of segments II and III combined, gill II as long as length of segments III and $2 / 3$ of IV combined, gill III as long as length of segments IV and V combined, gill IV as long as length of segments V and VI combined, gill V as long as length of segments VI and VII combined, gill VI as long as length of segments VII and VIII combined, gill VII as long as length of segments VIII to X combined.
\end{abstract}

PARAPRoct (Fig. 9I). Posterior margin with 10-14 stout spines; surface with scattered U-shaped scale bases; posterolateral extension (cercotractor) with numerous small, marginal spines.

Caudal filaments (Fig. 13A). Cerci ca $0.5 \times$ body length, median caudal filament ca. $0.8 \times$ length of cerci.

\title{
Distribution
}

Philippines: Luzon, Mindoro (Fig. 14C).

\section{Remarks}

The specimens were collected at low altitudes from 140 to $240 \mathrm{~m}$ a.s.l. in meandering alluvial rivers of small to medium size (width 2-12 m, depth $15-30 \mathrm{~cm}$, water current $0.08-0.80 \mathrm{~m} / \mathrm{s}$, temperature $23-28.7^{\circ} \mathrm{C}, \mathrm{pH} 6.8-8.3$, dissolved oxygen $\left.3.8-8.3 \mathrm{mg} / \mathrm{l}\right)$. The streams are surrounded by secondary vegetation, rarely secondary forest, with a few houses and farmland at some distance from the river bed as described in Garces et al. 2018.

\section{Genetics}

COI sequences were obtained from all three of the new species (Table 1). The genetic distances between these species are between $13 \%$ and $20 \%$, much higher than $3.5 \%$, which is generally considered as a likely maximal value for intraspecific divergence (Hebert et al. 2003; Ball et al. 2005; Zhou et al. 2010) (Table 4). Very limited genetic distances between 0 and $1 \%$ were found between specimens of the same species.

\section{Discussion}

Procerobaetis gen. nov. obviously belongs to the family Baetidae based on the developing turban eyes of late instar male larvae (Figs 12C-D, 13A-B), the long, slender, pisciform body shape, the larval Y-shaped frontal suture reaching ventrally of the lateral ocelli (Fig. 6M), the labrum with distinctly expressed median incision, the shape of the left and right prostheca, the largely fused outer and inner sets of denticles of both mandibles, the shape of the glossae (basally widened, most part narrow) and the anterior outer projection of the femur apex, which is directed toward the inner side of the femur (Wang \& 


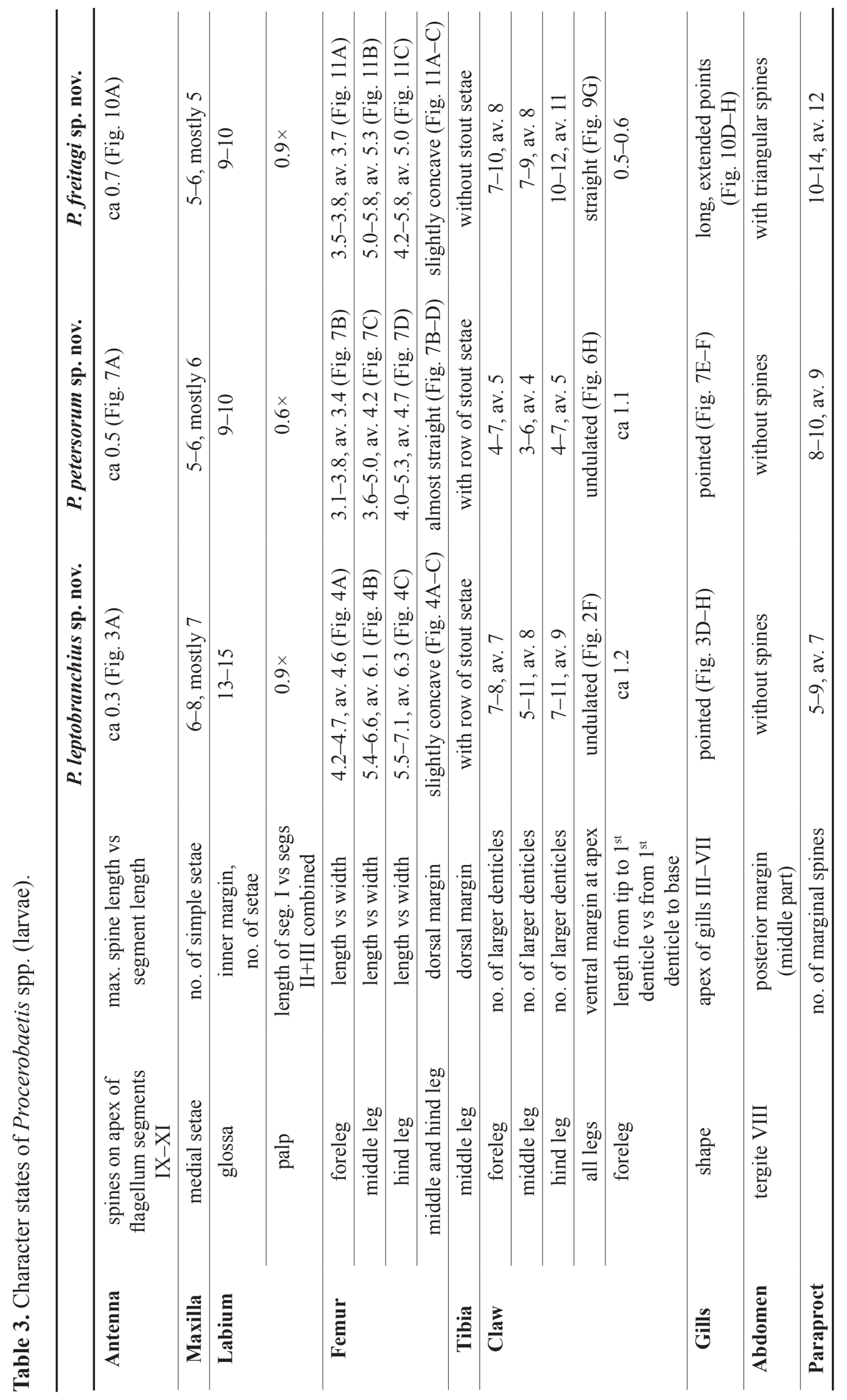


Table 4. Genetic distances (COI) between sequenced specimens, using the Kimura 2-parameter.

\begin{tabular}{cccccc}
\hline & & $\mathbf{1}$ & $\mathbf{2}$ & $\mathbf{3}$ & $\mathbf{4}$ \\
$\mathbf{1}$ & Procerobaetis leptobranchius sp. nov. & & & & \\
$\mathbf{2}$ & Procerobaetis petersorum sp. nov. & 0.13 & & & \\
$\mathbf{3}$ & Procerobaetis petersorum sp. nov. & 0.13 & 0.01 & & \\
$\mathbf{4}$ & Procerobaetis sp. C & 0.16 & 0.16 & 0.16 & \\
$\mathbf{5}$ & Procerobaetis freitagi sp. nov. & 0.20 & 0.18 & 0.19 & 0.20 \\
\hline
\end{tabular}

McCafferty 1996; Kluge 2004; Kluge \& Novikova 2011). It further belongs to the subfamily Baetinae sensu Kazlauskas (1972) and more specifically to the non-Baetis complex of Baetinae (Waltz et al. 1994; Waltz \& McCafferty 1997), also referred to as Baetungulata-non-Baetofemorata (Kluge \& Novikova 2011), because of the claw with one row of denticles on the inner-anterior side and the lack of a femoral patch. However, Procerobaetis gen. nov. has a 3-segmented maxillary palp, contrary to most Baetinae. The third segment is always rather short and the separation from the second segment is not always completely visible (Figs 1G, 5G, 8G); it therefore could be a recent, secondary division of segment II. Similar 3-segmented maxillary palps are also present in two species of Labiobaetis Novikova \& Kluge, 1987, from Indonesia (Kaltenbach \& Gattolliat 2019).

Procerobaetis gen. nov. is closely related to Nigrobaetis Kazlauskas in Novikova \& Kluge, 1987 and Alainites Waltz \& McCafferty, 1994. They share the typical rounded, involute appearance of the head and thorax in lateral view, the labium, including the shape of the glossae and paraglossae and the labial palp with a very small distolateral extension of segment II, and the labrum with long, stout, simple setae on the dorsal surface, which are erratically distributed and not arranged in an arc (Müller-Liebenau 1984; Waltz et al. 1994; Kang et al. 1994; Gattolliat 2004; Gattolliat et al. 2012; Zrelli et al. 2012). Nigrobaetis and Alainites are different by the shape of the gills, which are oval to elongate oval, whereas Procerobaetis gen. nov. is characterized by very slender, elongate gills with pronounced points and with a very long, extended apex at least in gills I and II (Figs 3B-H, 10B-H). This shape of gills I and II is an apomorphy, which is unique in Baetidae. However, similar gills are known from Leptophlebiidae as well (Peters \& Edmunds 1964, 1970). Furthermore, Alainites and Nigrobaetis can be distinguished by the 2-segmented maxillary palp, the absence of a medial tuft of stout, long setae on the hypopharynx, the absence of laterally pectinate setae on the legs, the shape of the claws (more extended and only slightly bent in Procerobaetis gen. nov.) and the presence of spines at the distal margin of abdominal tergite IV (Müller-Liebenau 1984, 1985; Kang et al. 1994; Gattolliat 2004; Gattolliat et al. 2012; Zrelli et al. 2012). Additionally, Alainites is different from Procerobaetis gen. nov. by the bifurcate left prostheca and the presence of a pronounced prolongation on the posterior side of the paraproct (MüllerLiebenau 1984; Zrelli et al. 2012), and Nigrobaetis is different by the shape of the left prostheca (more stout, with apical denticles) (Müller-Liebenau 1984; Gattolliat 2004; Gattolliat et al. 2012). Recently, Alainites was tentatively synonymized with Takobia Novikova \& Kluge, 1987 (Kluge \& Novikova 2014). Takobia maxillaris (Braasch \& Soldan, 1983), the type species of Takobia, has a labial palp and right mandible very similar to those of Procerobaetis gen. nov., but differs in many other characters, such as the maxillary palp, the claw without denticles, the tergites with spines at the posterior margin, the spines of the paraproct and the shape of the gills (Novikova \& Kluge 1987).

Some of the characters of Procerobaetis gen. nov. are also present in other, not closely related genera of Baetidae. Indocloeon Müller-Liebenau, 1982 has a similar left mandible with a tuft of setae and minute denticles between prostheca and mola, both directed toward the subtriangular process, a tuft of setae between the prostheca and mola of the right mandible, a tuft of stout setae medially on the 

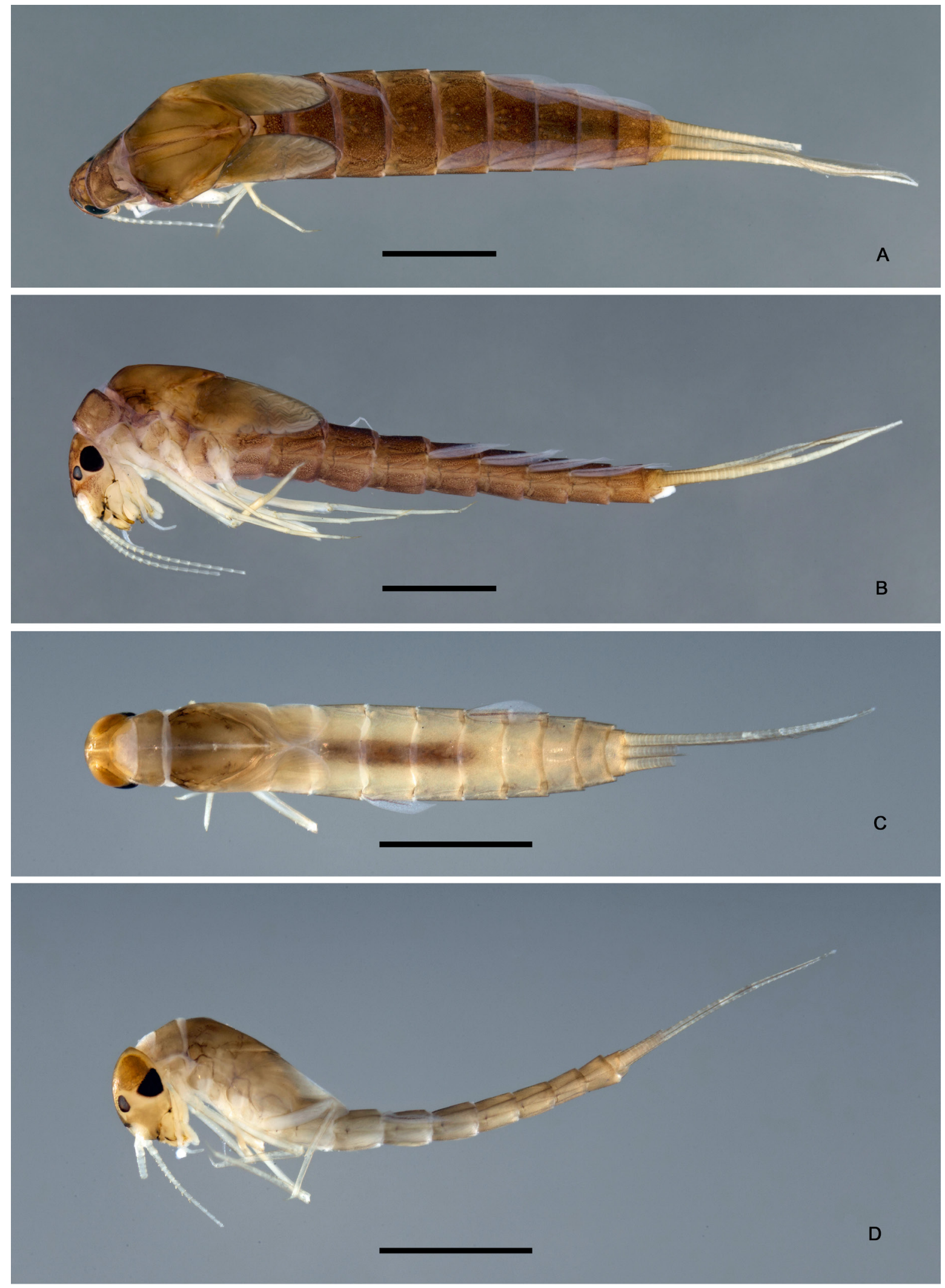

Fig. 12. Habitus, larvae. A. Procerobaetis leptobranchius gen. et sp. nov., dorsal view. B. P. leptobranchius gen. et sp. nov., lateral view. C. P. petersorum gen. et sp. nov., dorsal view. D. P. petersorum gen. et sp. nov., lateral view. Scale bars: $1 \mathrm{~mm}$. 

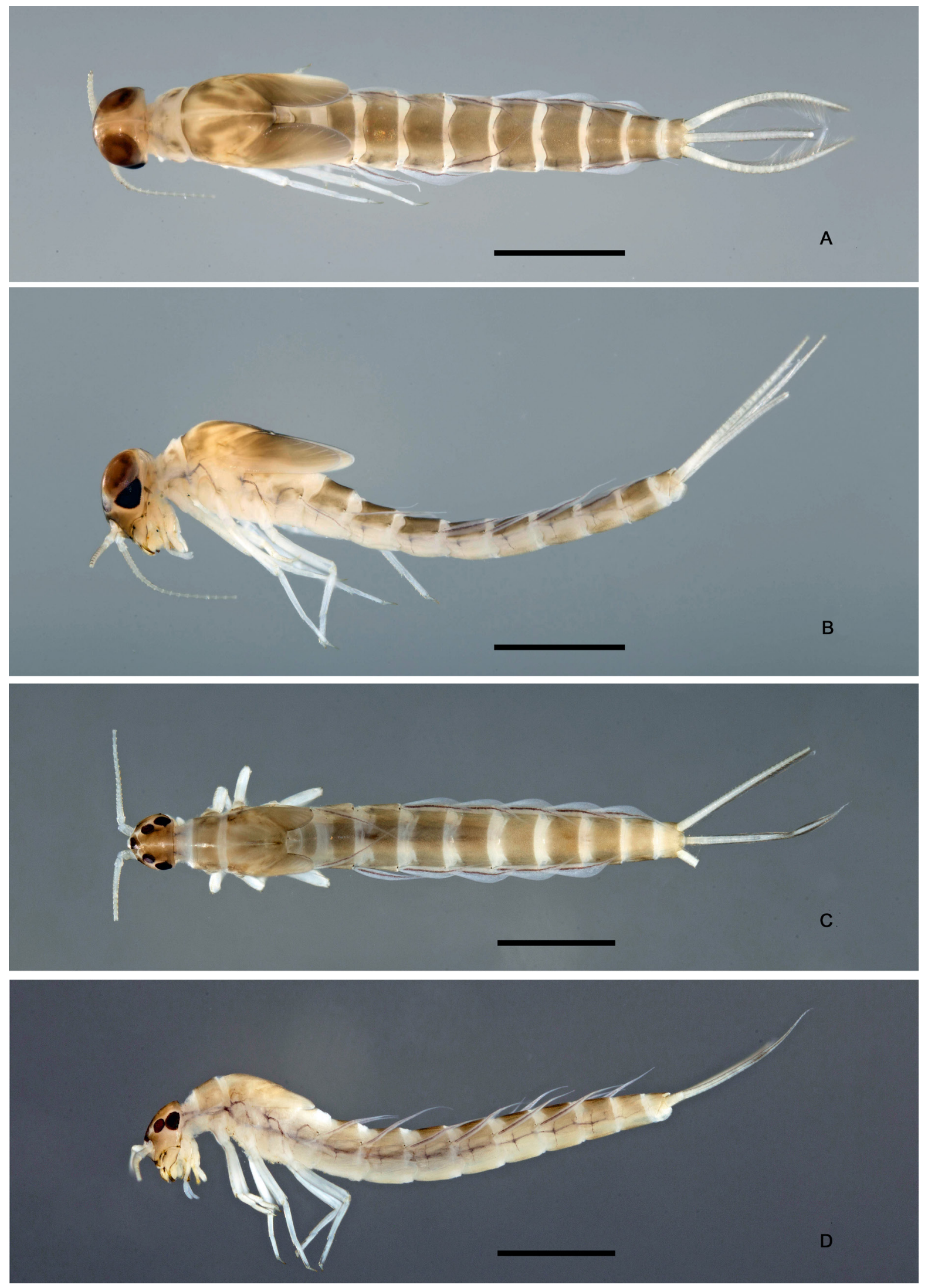

Fig. 13. Procerobaetis freitagi gen. et sp. nov., habitus, larvae. A. Dorsal view, Luzon. B. Lateral view, Luzon. C. Dorsal view, Mindoro. D. Lateral view, Mindoro. Scale bars: $1 \mathrm{~mm}$. 
hypopharynx, and legs with laterally pectinate setae on femur, tibia and tarsus; moreover, Indocloeon spathasetis Kaltenbach \& Gattolliat, 2017 has similar spines on the outer lateral margin of the flagellum (Kluge 2012; Kaltenbach \& Gattolliat 2017). It is distinguished by the labial palp having a distomedial protuberance on segment II, the claw with two rows of denticles, the foreleg lacking a patellotibial suture (Protopatellata), the spines at the posterior margins of the tergites and the shape of the gills (Kluge 2012; Kaltenbach \& Gattolliat 2017). Cheleocloeon Wuillot \& Gillies, 1993 shares similar left and right mandibles and the elongate, slender, slightly bent claws. It differs by the two rows of denticles on the claws (in some species extremely reduced or vistigial), the labial palp with a large distomedial protuberance of segment II, the lack of a tuft of stout setae on the hypopharynx, the spines on the posterior margin of the tergites and the shape of the gills (Lugo-Ortiz \& McCafferty 1997; Gattolliat \& Sartori 2008; Kluge 2016).
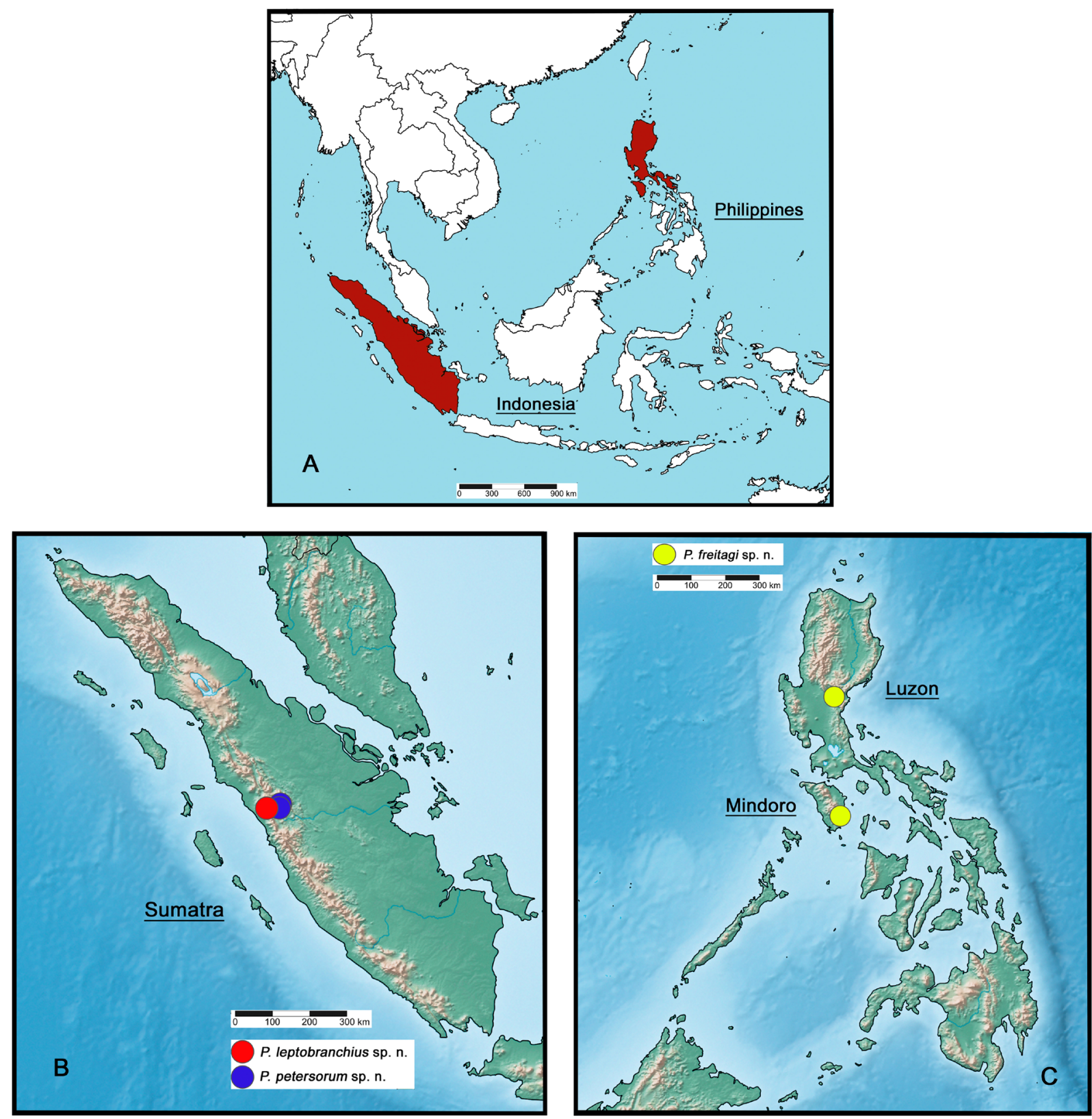

Fig. 14. Distribution of Procerobaetis gen. nov. A. Map of Southeast Asia. B. Localities in Indonesia. C. Localities in the Philippines. 
The genetic distances between the different species of Procerobaetis gen. nov. are in an expected range, between 13 and $20 \%$ (K2P, Table 4). Ball et al. (2005) reported a mean interspecific, congeneric distance of $18 \%$ for mayflies in the USA and Canada. The intraspecific distances are very low, as expected, ranging from 0 to $1 \%(\mathrm{~K} 2 \mathrm{P})$. This result is certainly biased, as it is based on two sequenced specimens per species from a single population.

Interestingly, we found two different species, Procerobaetis leptobranchius gen. et sp. nov. and P. petersorum gen. et sp. nov., in the same area in Sumatra (Fig. 14B). However, they were found on the slopes of two different volcanos, at different elevations and under different ecological conditions. Procerobaetis leptobranchius gen. et sp. nov. was collected on the volcano Singgalang at altitudes from 1600 to $1800 \mathrm{~m}$ a.s.l. ( $\mathrm{pH} \mathrm{6-7,} \mathrm{water} \mathrm{temperature} 15.5-17^{\circ} \mathrm{C}$ ), while P. petersorum gen. et sp. nov. was collected on the volcano Sago at altitudes from 880 to $1100 \mathrm{~m}$ a.s.1. $(\mathrm{pH} 7-7.5$, water temperature $20-21{ }^{\circ} \mathrm{C}$ ). Furthermore, we have a single, small larva of Procerobaetis gen. nov. from another neighboring volcano, Marapi, collected at an altitude of $1400 \mathrm{~m}$ a.s.l. This $P$. sp. C has a genetic distance of $16 \%$ to the other two species from Sumatra, which is clearely in the interspecific range (Table 4). We are unable to establish resilient morphological differences between $P$. sp. C and the other species based on a single small larva; therefore, $P$. sp. $\mathrm{C}$ will remain an undescribed species for now, without further treatment in this paper. However, the occurrence of at least two different species in the same area at different elevations and ecological conditions points to the direction that these factors could be drivers of speciation by radiation. In a recent study of the structuration of the mayfly community in the same area (volcanos Singgalang, Sago and Marapi in Sumatra), elevation was found to be the only factor driving the within-species genetic structuring of two species of Baetidae and an important factor for the two others (Gueuning et al. 2017).

Procerobaetis gen. nov. has a wide distribution across Southeast Asia (Indonesia, Philippines). However, our knowledge about Baetidae in this region is still poor and no collections were done at all in many areas or habitats. Therefore, we expect more species of this genus in Southeast Asia with further collections in the future.

\section{Acknowledgements}

We sincerely thank Morgan Gueuning (University of Neuchâtel, Switzerland), who collected the material from Sumatra during a Master's project at the University of Lausanne (UNIL) and the Museum of Zoology Lausanne (MZL). Collection in the Philippines was made possible by a Gratuitous Permit (GP 0133-17 and renewals) for the collection of aquatic wildlife in parts of Mindoro and Luzon, as kindly issued by the Bureau of Fisheries and Aquatic Resources, Quezon City. Fieldwork and management of aquatic macroinvertebrate materials in the Biodiversity Laboratory was partly supported by the School of Science and Engineering Industry 4.0 Research Fund (SI4-013), AdMU. The graduate studies of Jhoana M. Garces are supported by the Advanced Science and Technology Human Resource Development Program of the Department of Science and Technology (ASTHRDP-DOST). Furthermore, we are grateful to Michel Sartori (MZL) for his interest and support for our project and to Marion Podolak (MZL) for her always dedicated technical assistance. Finally, we are thankful to Cassandra Caedo (USA) for corrections and improvements of the English language, and to the reviewers of our manuscript for their valuable comments.

\section{References}

Ball S.L., Hebert P.D.N., Burian S.K. \& Webb J.M. 2005. Biological identifications of mayflies (Ephemeroptera) using DNA barcodes. Journal of the North American Benthological Society 24: 508524. https://doi.org/10.1899/04-142.1

Brown R.M. \& Diesmos A.C. 2010. Philippines, Biology. In: Gillespie R.G. \& Clague D.A. (eds) Encyclopedia of Islands: 723-732. University of California Press, Berkeley, Los Angeles, London. 
Chakrabarty P., Warren M., Page L.M. \& Baldwin C.C. 2013. GenSeq: An updated nomenclature and ranking for genetic sequences from type and non-type sources. Zookeys 346: $29-41$. https://doi.org/10.3897/zookeys.346.5753

Folmer O., Black M., Hoeh W., Lutz R. \& Vrijenhoek R. 1994. DNA primers for amplification of mitochondrial cytochrome c oxidase subunit I from diverse metazoan invertebrates. Molecular Marine Biology and Biotechnology 3: 294-299.

Available from http://www.mbari.org/staff/vrijen/PDFS/Folmer_94MMBB.pdf [accessed 13 Feb. 2020].

Freitag H., Jäch M.A. \& Wewalka G. 2016. Diversity of aquatic and riparian Coleoptera of the Philippines: checklist, state of knowledge, priorities for future research and conservation. Aquatic Insects 37: 177213. https://doi.org/10.1080/01650424.2016.1210814

Garces J.M., Bauernfeind E. \& Freitag H. 2018. Sparsorythus sescarorum, new species from Mindoro, Philippines (Ephemeroptera, Tricorythidae). ZooKeys 795: 13-30.

https://doi.org/10.3897/zookeys.795.28412

Gattolliat J.-L. 2004. First records of the genus Nigrobaetis Novikova \& Kluge (Ephemeroptera: Baetidae) from Madagascar and La Réunion with observations on Afrotropical biogeography. Revue Suisse de Zoologie 111 (3): 657-669.

Available from https://biodiversitylibrary.org/page/41187547 [accessed 13 Feb. 2020].

Gattolliat J.-L. 2012. Two new genera of Baetidae (Ephemeroptera) from Borneo (East Kalimantan, Indonesia). International Journal of Limnology 48: 187-199. https://doi.org/10.1051/limn/2012012

Gattolliat J.-L. \& Nieto C. 2009. The family Baetidae (Insecta: Ephemeroptera): synthesis and future challenges. Aquatic Insects 31: 41-62. https://doi.org/10.1080/01650420902812214

Gattolliat J.-L. \& Sartori M. 2008. Order Ephemeroptera. Arthropod Fauna of the UAE 1: 47-83.

Gattolliat J.-L., Vuataz L. \& Sartori M. 2012. First contribution to the mayflies of Jordan (Insecta: Ephemeroptera). Zoology in the Middle East 56: 91-110.

https://doi.org/10.1080/09397140.2012.10648945

Gueuning M., Suchan T., Rutschmann S., Gattolliat J.-L., Jamsari J., Kamil A.I., Pitteloud C., Buerki S., Balke M., Sartori M. \& Alvarez N. 2017. Elevation in tropical sky islands as the common driver in structuring genes and communities of freshwater organisms. Scientific Reports 7: 16089-16103.

https://doi.org/10.1038/s41598-017-16069-y

Hall R. 2010. Indonesia, Geology. In: Gillespie R.G. \& Clague D.A. (eds) Encyclopedia of Islands: 454-460. University of California Press, Berkeley, Los Angeles, London.

Hebert P.D.N., Cywinska A., Ball S.L. \& DeWaard J.R. 2003. Biological identifications through DNA barcodes. Proceedings of The Royal Society B 270: 313-321. https://doi.org/10.1098/rspb.2002.2218

Hubbard M.D. 1995. Towards a standard methodology for the description of mayflies (Ephemeroptera). In: Corkum L.D. \& Ciborowski J.J.H. (eds) Current Directions in Research on Ephemeroptera: 361369. Canadian Scholar's Press, Toronto.

Jacobus L.M., Macadam C.R. \& Sartori M. 2019. Mayflies (Ephemeroptera) and their contributions to ecosystem services. Insects 10: 1-26. https://doi.org/10.3390/insects10060170

Kaltenbach T. \& Gattolliat J.-L. 2017. New species of Indocloeon Müller-Liebenau from South-East Asia (Ephemeroptera, Baetidae). Zookeys 723: 43-60. https://doi.org/10.3897/zookeys.723.20578

Kaltenbach T. \& Gattolliat J.-L. 2019. The tremendous diversity of Labiobaetis Novikova \& Kluge in Indonesia (Ephemeroptera, Baetidae). Zookeys 895: 1-117. https://doi.org/10.3897/zookeys.895.38576

Kang C.-H., Chang H.-C. \& Yang C.-T. 1994. A revision of the genus Baetis in Taiwan (Ephemeroptera, Baetidae). Journal of Taiwan Museum 47 (2): 9-44. [In Chinese] 
Kazlauskas R.S. 1972. Neues über das System der Eintagsfliegen der Familie Baetidae (Ephemeroptera). Proceedings of the XIII International Congress of Entomology in Moscow, 2-9 August 1968 3:337-338.

Kimura M. 1980. A simple method for estimating evolutionary rates of base substitutions through comparative studies of nucleotide sequences. Journal of Molecular Evolution 16: 111-120.

https://doi.org/10.1007/BF01731581

Kingston T. 2010. Indonesia, Biology. In: Gillespie R.G. \& Clague D.A. (eds) Encyclopedia of Islands: 446-453. University of California Press, Berkeley, Los Angeles, London.

Kluge N. 2004. The Phylogenetic System of Ephemeroptera. Kluwer Academic Publishers, Dordrecht, the Netherlands.

Kluge N.J. 2012. Non-African representatives of the plesiomorphion Protopatellata (Ephemeroptera: Baetidae). Russian Entomological Journal 20: 361-376.

Kluge N.J. 2016. Redescription of the genus Cheleocloeon Wuillot \& Gillies 1993 (Ephemeroptera: Baetidae) with descriptions of three new species from Zambia and Uganda. Zootaxa 4067: 135-167. https://doi.org/10.11646/zootaxa.4067.2.2

Kluge N.J. \& Novikova E.A. 2011. Systematics of the mayfly taxon Acentrella (Ephemeroptera, Baetidae), with description of new Asian and African species. Russian Entomological Journal 20: 1-56.

Kluge N.J. \& Novikova E.A. 2014. Systematics of Indobaetis Müller-Liebenau \& Morihara 1982, and related implications for some other Baetidae genera (Ephemeroptera). Zootaxa 3835: 209-236.

https://doi.org/10.11646/zootaxa.3835.2.3

Kumar S., Stecher G. \& Tamura K. 2016. MEGA 7: Molecular Evolutionary Genetics Analysis version 7.0 for bigger datasets. Molecular Biology and Evolution 33: 1870-1874.

https://doi.org/10.1093/molbev/msw054

Lugo-Ortiz C.R. \& McCafferty W.P. 1997. Contribution to the systematics of the genus Cheleocloeon (Ephemeroptera: Baetidae). Entomological News 108: 283-289.

Morihara D.K. \& McCafferty W.P. 1979. The Baetis larvae of North America (Ephemeroptera: Baetidae). Transactions of the American Entomological Society 105: 139-221.

Available from https://www.jstor.org/stable/25078238 [accessed $17 \mathrm{Feb} .2020$ ].

Müller-Liebenau I. 1984. New genera and species of the family Baetidae from West-Malaysia (River Gombak) (Insecta: Ephemeroptera). Spixiana 7: 253-284.

Available from https://biodiversitylibrary.org/page/28279448 [accessed 17 Feb. 2020].

Müller-Liebenau I. 1985. Baetidae from Taiwan with remarks on Baetiella Ueno, 1931 (Insecta, Ephemeroptera). Archiv für Hydrobiologie 104: 93-110.

Novikova E.A. \& Kluge N.J. 1987. Systematics of the genus Baetis (Ephemeroptera, Baetidae), with descriptions of new species from Middle Asia. Vestnik Zoologii 1987(4): 8-19. [In Russian]

Odgen T.H. \& Whiting M.F. 2005. Phylogeny of Ephemeroptera (mayflies) based on molecular evidence. Molecular Phylogenetics and Evolution 37: 625-643. https://doi.org/10.1016/j.ympev.2005.08.008

Ogden T.H., Gattolliat J.-L., Sartori M., Staniczek A.H., Soldan T. \& Whiting M.F. 2009. Towards a new paradigm in mayfly phylogeny (Ephemeroptera): combined analysis of morphological and molecular data. Systematic Entomology 34: 616-634. https://doi.org/10.1111/j.1365-3113.2009.00488.x

Peters W.L. \& Edmunds G.F. Jr. 1964. A revision of the generic classification of the Ethiopian Leptophlebiidae (Ephemeroptera). Transactions of the Royal Entomological Society of London 116: 225-253.

Peters W.L. \& Edmunds G.F. Jr. 1970. Revision of the generic classification of the Eastern Hemisphere Leptophlebiidae (Ephemeroptera). Pacific Insects 12: 157-240. 
Sanger F., Nicklen S. \& Coulson A.R. 1977. DNA sequencing with chain-terminating inhibitors. Proceedings of the NationalAcademy of Sciences 74: 5463-5467.https://doi.org/10.1073/pnas.74.12.5463

Sartori M. \& Brittain J.E. 2015. Order Ephemeroptera. In: Thorp J. \& Rogers D.C. (eds) Ecology and General Biology: Thorp and Corvich's Freshwater Invertebrates: 873-891. Academic Press, Boston. https://doi.org/10.1016/B978-0-12-385026-3.00034-6

Sartori M., Derleth P. \& Gattolliat J.-L. 2003. New data about the mayflies (Ephemeroptera) from Borneo. In: Gaino E. (ed.) Research Update on Ephemeroptera and Plecoptera: 403-406. University of Perugia, Perugia, Italy.

Shorthouse D.P. 2010. SimpleMappr, an online tool to produce publication-quality point maps. Available from https://www.simplemappr.net [accessed 3 Jul. 2019].

Vuataz L., Sartori M., Wagner A. \& Monaghan M.T. 2011. Toward a DNA taxonomy of Alpine Rhithrogena (Ephemeroptera: Heptagenidae) using a mixed Yule-coalescent analysis of mitochondrial and nuclear DNA. PLoS ONE 6: 1-11. https://doi.org/10.1371/journal.pone.0019728

Waltz R.D. \& McCafferty W.P. 1997. New generic synonymies in Baetidae (Ephemeroptera). Entomological News 108: 134-140.

Waltz R.D., McCafferty W.P. \& Thomas A. 1994. Systematics of Alainites n. gen., Diphetor, Indobaetis, Nigrobaetis n. stat., and Takobia n. stat. (Ephemeroptera, Baetidae). Bulletin de la Societé d'Histoire naturelle de Toulouse 130: 33-36.

Wang T.-Q. \& McCafferty W.P. 1996. New diagnostic characters for the mayfly family Baetidae (Ephemeroptera). Entomological News 107: 207-212.

Zhou X., Jacobus L.M., DeWalt R.E., Adamowicz S.J. \& Hebert P.D.N. 2010. Ephemeroptera, Plecoptera, and Trichoptera fauna of Churchill (Manitoba, Canada): insights into biodiversity patterns from DNA barcoding. Journal of the North American Benthological Society 29: 814-837.

https://doi.org/10.1899/09-121.1

Zrelli S., Gattolliat J.-L., Boumaïza M. \& Thomas A. 2012. First record of Alainites sadati Thomas, 1994 (Ephemeroptera: Baetidae) in Tunisia, description of the larval stage and ecology. Zootaxa 3497 (1): 60-68. https://doi.org/10.11646/zootaxa.3497.1.6

Manuscript received: 18 September 2019

Manuscript accepted: 25 November 2019

Published on: 9 March 2020

Topic editor: Gavin Broad

Desk editor: Danny Eibye-Jacobsen

Printed versions of all papers are also deposited in the libraries of the institutes that are members of the EJT consortium: Muséum national d'histoire naturelle, Paris, France; Meise Botanic Garden, Belgium; Royal Museum for Central Africa, Tervuren, Belgium; Royal Belgian Institute of Natural Sciences, Brussels, Belgium; Natural History Museum of Denmark, Copenhagen, Denmark; Naturalis Biodiversity Center, Leiden, the Netherlands; Museo Nacional de Ciencias Naturales-CSIC, Madrid, Spain; Real Jardín Botánico de Madrid CSIC, Spain; Zoological Research Museum Alexander Koenig, Bonn, Germany; National Museum, Prague, Czech Republic. 